\title{
Inverse subsumption for complete explanatory induction
}

\author{
Yoshitaka Yamamoto • Katsumi Inoue · Koji Iwanuma
}

Received: 27 July 2010 / Accepted: 19 April 2011 / Published online: 7 July 2011

(C) The Author(s) 2011

\begin{abstract}
Modern explanatory inductive logic programming methods like Progol, Residue procedure, CF-induction, HAIL and Imparo use the principle of inverse entailment (IE). Those IE-based methods commonly compute a hypothesis in two steps: by first constructing an intermediate theory and next by generalizing its negation into the hypothesis with the inverse of the entailment relation. Inverse entailment ensures the completeness of generalization. On the other hand, it imposes many non-deterministic generalization operators that cause the search space to be very large. For this reason, most of those methods use the inverse relation of subsumption, instead of entailment. However, it is not clear how this logical reduction affects the completeness of generalization. In this paper, we investigate whether or not inverse subsumption can be embedded in a complete induction procedure; and if it can, how it is to be realized. Our main result is a new form of inverse subsumption that ensures the completeness of generalization. Consequently, inverse entailment can be reduced to inverse subsumption without losing the completeness for finding hypotheses in explanatory induction.
\end{abstract}

Keywords Inverse entailment - Inverse subsumption - Learning from entailment · Explanatory induction · Inductive logic programming

Editors: Paolo Frasconi and Francesca Lisi.

Y. Yamamoto $(\bowtie) \cdot K$. Iwanuma

University of Yamanashi, 4-3-11 Takeda, Kofu, Yamanashi 400-8510, Japan

e-mail: yyamamoto@yamanashi.ac.jp

K. Iwanuma

e-mail: iwanuma@yamanashi.ac.jp

K. Inoue

National Institute of Informatics, 2-1-2 Hitotsubashi, Chiyoda-ku, Tokyo 101-8430, Japan

e-mail: ki@nii.ac.jp 
Fig. 1 Hypothesis finding based on inverse entailment

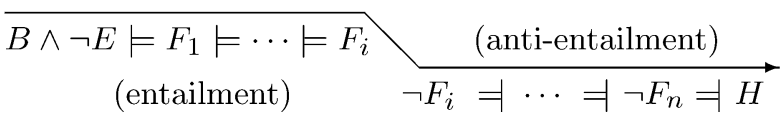

\section{Introduction}

Learning from entailment (Muggleton 1995; Flach 1996; De Raedt 1997; Inoue 2004) is one of the most widely studied frameworks for the paradigm of inductive machine learning. Given a background theory $B$ and examples $E$, the task of learning from entailment is to find a hypothesis $H$ such that $B \wedge H \models E$ where $B \wedge H$ is consistent. This style of inductive learning is alternatively called explanatory induction (Flach 1996) and is used as a standard setting in inductive logic programming (ILP) (Muggleton and De Raedt 1994; NienhuysCheng and De Wolf 1997). By the principle of inverse entailment (IE) (Muggleton 1995), the above task is logically equivalent to finding a consistent hypothesis $H$ such that $B \wedge \neg E \models$ $\neg H$. This equivalence means that the inductive hypothesis $H$ can be computed by deriving its negation $\neg H$ from $B$ and $\neg E$. We can represent this derivation process as:

$$
B \wedge \neg E \models F_{1} \models \cdots \models F_{i} \models \cdots \models F_{n} \models \neg H
$$

where each $F_{i}(1 \leq i \leq n)$ denotes a clausal theory.

Modern explanatory ILP methods like Progol (Muggleton 1995; Tamaddoni-Nezhad and Muggleton 2009), Residue procedure (Yamamoto 2003), CF-induction (Inoue 2004; Yamamoto et al. 2008), HAIL (Ray et al. 2003; Ray and Inoue 2008; Ray 2009) and Imparo (Kimber et al. 2009) are based on IE. These IE-based methods compute a hypothesis $H$ in two steps: by first constructing an intermediate theory $F_{i}$ in Relation (1) and next generalizing its negation $\neg F_{i}$ into the hypothesis $H$. The relation between $\neg F_{i}$ and $H$ can be obtained from the contrapositive of Relation (1) as:

$$
\neg(B \wedge \neg E)=\neg F_{1}=\cdots \Rightarrow \neg F_{i}=\cdots \Rightarrow \neg F_{n}=H
$$

where $=1$ denotes the inverse relation of entailment, simply called anti-entailment. ${ }^{1}$ In other words, every IE-based method first uses the entailment relation to construct $F_{i}$ in Relation (1), and then switches to anti-entailment to generate the hypothesis $H$ in Relation (2). (See Fig. 1.)

Inverse entailment ensures the completeness of generalization in the sense of generating any hypothesis $H$ such that $F_{i} \models \neg H$ for an intermediate theory $F_{i}$ in Fig. 1 . On the other hand, it needs a variety of different operators such as inverse resolution (Muggleton and Buntine 1988) which applies the inverse of the resolution principle. There are several such operators each of which can be applied in many different ways. This fact leads to a large number of choice points that cause the huge search space of IE-based methods. For this reason, some methods use the inverse relation of subsumption, simply called anti-subsumption, due to computational efficiency. However, it was not clear whether or not their generalization becomes incomplete by reducing anti-entailment to anti-subsumption, and thus they may fail to find a relevant hypothesis worth considering. To distinguish their specific approach using anti-subsumption from IE, we term it inverse subsumption (IS).

\footnotetext{
${ }^{1}$ We distinguish two terms: anti-entailment and inverse entailment. Note that inverse entailment generally indicates the approach to find hypotheses using anti-entailment in ILP.
} 
Fig. 2 Hypothesis finding based on inverse subsumption

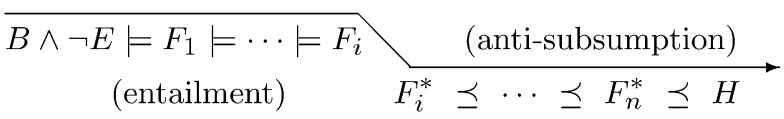

For this open problem, the paper investigates whether or not inverse subsumption can be embedded in a complete inductive procedure; and if it can, how it is to be realized. Consequently, the paper shows a new form of inverse subsumption that can ensure the completeness of generalization. Our result is applicable to every previously proposed method. Firstly, it enables to logically characterize the possible hypotheses obtained by each IS-based method (Muggleton 1995; Yamamoto 2003; Ray et al. 2003; Ray and Inoue 2008; Ray 2009; Tamaddoni-Nezhad and Muggleton 2009; Kimber et al. 2009). Secondly, it enables to logically simplify the generalization procedure in each IE-based method (Inoue 2004; Yamamoto et al. 2008) using the new form of inverse subsumption without losing the completeness for finding hypotheses.

The key idea lies in the logical relation $F_{i} \models \neg H$ for a ground intermediate theory $F_{i}$ and a ground hypothesis $H$ in Relation (1). We show that there is a certain clausal theory $F_{i}^{*}$ such that $F_{i}^{*}$ is logically equivalent to $\neg F_{i}$ and $F_{i}^{*} \preceq H$. Note here that $\preceq$ denotes antisubsumption, that is, $F_{i}^{*}$ is subsumed by $H$.

Example 1 We give the intuition of our idea by drawing a passage from Hamlet. Given $E_{1}=$ defeat(claudius), suppose the following hypothesis:

$$
H_{1}=\text { risk_life }(\text { hamlet }) \wedge(\text { risk_life }(\text { hamlet }) \supset \text { defeat }(\text { claudius })) .
$$

$H_{1}$ may describe Hamlet's decision to risk his life to defeat Claudius. Let an intermediate theory $F_{i}$ be $\neg E_{1}$. The complement $\overline{F_{i}}=\operatorname{defeat}$ (claudius) is entailed by $H_{1}$, but is not subsumed by it. We then consider another intermediate theory $F_{i}^{\prime}$ obtained by adding the tautology risk_life(hamlet) $\vee \neg$ risk_life(hamlet) to $F_{i}$. Since $F_{i}^{\prime}$ is logically equivalent to $F_{i}$, the following complement $\overline{F_{i}^{\prime}}$ :

\section{$($ risk_life $($ hamlet $) \vee$ defeat $($ claudius $)) \wedge($ risk_life $($ hamlet $) \supset$ defeat $($ claudius $))$}

is also entailed by $H_{1}$. Besides, $\overline{F_{i}^{\prime}}$ is subsumed by $H_{1}$, unlike the case of $\overline{F_{i}}$. Hence, adding the tautology (i.e. Hamlet risks his life or not) plays a role of reducing anti-entailment to anti-subsumption. Note that the above $F_{i}^{*}$ can be regarded as $\overline{F_{i}^{\prime}}$.

This feature enables us to logically reduce the derivation process based on inverse entailment in Fig. 1 to the one based on inverse subsumption described in Fig. 2.

This paper uses two kinds of clausal theories regarded as $F_{i}^{*}$, called residue and minimal complements, respectively. Together with these two complements, we show how inverse entailment can be reduced to inverse subsumption. In both cases, inverse subsumption is sufficient to ensure the completeness of generalization.

The rest of this paper is organized as follows. After Sect. 2 describes the theoretical background, we show inverse subsumption with residue and minimal complements ensure the completeness of generalization in Sects. 3 and 4, respectively. In Sect. 5, we clarify some commonness between two approaches with residue and minimal complements and apply them to each IE-based method. In Sect. 6, we conclude. 


\section{Background}

\subsection{Preliminaries}

We assume the reader to be familiar with the basic concepts in first-order logic and inductive logic programming (Nienhuys-Cheng and De Wolf 1997). A clause is a finite disjunction of literals which is often identified with the set of its literals. A clause of the form $\left\{\neg B_{1}, \ldots, \neg B_{n}, A_{1}, \ldots, A_{m}\right\}$, where each $A_{i}, B_{j}$ is an atom, is also written as $B_{1} \wedge \cdots \wedge B_{n} \supset A_{1} \vee \cdots \vee A_{m}$. Every variable of a clause is assumed to be universally quantified at the front. A Horn clause is a clause which contains at most one positive literal; otherwise it is a non-Horn clause. It is known that a clause is a tautology if it has two complementary literals $A$ and $\neg A$. We denote by $\perp$ the empty clause which contains no literal. Note that $\perp$ is inconsistent.

A clausal theory is a finite set of clauses, which represents the conjunction of the clauses in it. A clausal theory is full if it contains at least one non-Horn clause. A clausal theory is ground if it contains no variable. A (universal) conjunctive normal form (CNF) formula is a conjunction of clauses, and a disjunctive normal form (DNF) formula is a disjunction of conjunctions of literals. A clausal theory is identified with the CNF formula that is the conjunction of clauses in it. We denote by $\models$ the classical logical entailment relation and by $=$ the inverse relation of entailment, called anti-entailment. Let $S$ and $T$ be two clausal theories. $S$ and $T$ are (logically) equivalent, denoted by $S \equiv T$, if $S \models T$ and $T \models S$. A clause $C$ is a consequence of $S$ if $S \models C$.

A (ground) substitution $\theta$ replaces variables $x_{1}, \ldots, x_{k}$ occurring in a clause $C$ to (ground) terms $t_{1}, \ldots, t_{k}$ in $C \theta$. Note that $C \theta$ is called an instance of $C$. Let $L_{1}$ and $L_{2}$ be two literals. A substitution $\theta$ is called a unifier for $L_{1}$ and $L_{2}$ if $L_{1} \theta=L_{2} \theta$. A unifier $\theta$ is a most general unifier (mgu) if there is no other unifier $\sigma$ for which the unified literal $L_{1} \sigma$ is more general than $L_{1} \theta$. Let $C$ and $D$ be two clauses. $C$ subsumes $D$, denoted by $C \succeq D$, if there is a substitution $\theta$ such that $C \theta \subseteq D$. C properly subsumes $D$ if $C \succeq D$ but $D \nsucceq C$.

Definition 1 (Theory-subsumption) Let $S$ and $T$ be two clausal theories. Then, $S$ (theory-) subsumes $T$, denoted by $S \succeq T$, if for any clause $D \in T$, there is a clause $C \in S$ such that $C \succeq D$. We denote by $\preceq$ the inverse relation of the (theory-) subsumption, called antisubsumption.

Now, we have two concepts: entailment and subsumption to characterize the logical relation between two clausal theories $S$ and $T$. It is known that $S \models T$ holds if $S \succeq T$, though $S \succeq T$ does not necessarily hold even if $S \models T$. Suppose two clausal theories $S=\{p(X) \supset p(f(X))\}$ and $T=\{p(Y) \supset p(f(f(Y)))\}$ are given. Indeed, $S \nsucceq T$, but $S \models T$ holds, since $T$ is derivable from $S$ using the resolution principle. The two concepts can be logically connected in the context of the resolution principle by Lee's theorem (Lee 1967), alternatively called the Subsumption theorem (Nienhuys-Cheng and De Wolf 1997).

Let $C$ and $D$ (called parent clauses) be two clauses, and $L_{c}$ and $L_{d}$ two literals in $C$ and $D$, respectively. If there is a most general unifier $\theta$ for $L_{c}$ and $\neg L_{d}$, then the clause

$$
\left(C \theta-\left\{L_{c} \theta\right\}\right) \cup\left(D \theta-\left\{L_{d} \theta\right\}\right)
$$

is called a resolvent of $C$ and $D$. For example, recall the above $S$ and $T$. The clause in $T$ is a resolvent of two copies of the clause in $S: R_{1}=p\left(X_{1}\right) \supset p\left(f\left(X_{1}\right)\right)$ and $R_{2}=p\left(X_{2}\right) \supset$ $p\left(f\left(X_{2}\right)\right)$, since two literals $p\left(f\left(X_{1}\right)\right)$ and $p\left(X_{2}\right)$ has a mgu $\theta$ that replaces $X_{1}$ and $X_{2}$ to 
$Y$ and $f(Y)$, respectively. Then, the resolvent $\left(R_{1} \theta-\{p(f(Y))\}\right) \cup\left(R_{2} \theta-\{\neg p(f(Y))\}\right)$ corresponds to the clause in $T$.

A derivation of a clause $C$ from $S$ is a finite sequence of clauses $R_{1}, \ldots, R_{k}=C$ such that each $R_{i}$ is either in $S$, or is a resolvent of two clauses in $\left\{R_{1}, \ldots, R_{i-1}\right\}$. Then, the Subsumption theorem states that $S \models T$ if and only if for each clause $C \in T, C$ is a tautology or there is a derivation of a clause $D$ from $S$ such that $D$ subsumes $C$.

\subsection{Hypothesis finding based on inverse entailment}

We give the definition of a hypothesis $H$ in the setting of learning from entailment:

Definition 2 (Hypothesis) Let $B$ and $E$ be clausal theories, representing a background theory and positive examples, respectively. A clausal theory $H$ is a hypothesis wrt $B$ and $E$ if $H$ satisfies that $B \wedge H \models E$ and $B \wedge H$ is consistent.

We refer to a hypothesis instead of a hypothesis wrt $B$ and $E$ if no confusion arises.

Example 2 Suppose that

$$
B_{2}=\{\text { buy }(\text { john, diaper }) \vee \operatorname{buy}(\text { john, beer })\}, \quad E_{2}=\{\operatorname{shopping}(\text { john, at_night })\}
$$

are given. Then,

$$
\begin{aligned}
H_{2}=\{\text { buy }(X, \text { diaper }) & \supset \operatorname{buy}(X, \text { beer }), \\
\text { buy }(Y, \text { beer }) & \supset \operatorname{shopping}(Y, \text { at_night })\}
\end{aligned}
$$

is a hypothesis, since $B_{2} \wedge H_{2} \models E_{2}$ and $B_{2} \wedge H_{2}$ is consistent. Note here that the clause (3) means that customers who buy diapers also tend to buy beer, ${ }^{2}$ and the clause (4) means that customers who buy beer tend to go shopping at night.

Hypothesis finding in Definition 2 is logically equivalent to seeking a consistent hypothesis $H$ such that $B \wedge \neg E \models \neg H$. Using this alternative condition, IE-based methods (Muggleton 1995; Yamamoto 2003; Ray et al. 2003; Ray and Inoue 2008; Ray 2009; Tamaddoni-Nezhad and Muggleton 2009; Kimber et al. 2009) compute a hypothesis $H$ in two steps. First, they construct an intermediate theory $F$ such that $F$ is ground and $B \wedge \neg E \models F$. Hereafter, we call $F$ a bridge theory wrt $B$ and $E$ as follows.

Definition 3 (Bridge theory) Let $B$ and $E$ be a background theory and examples, respectively. Let $F$ be a ground clausal theory. Then $F$ is a bridge theory wrt $B$ and $E$ if $B \wedge \neg E \models F$ holds. If no confusion arises, a bridge theory wrt $B$ and $E$ will simply be called a bridge theory.

After constructing a bridge theory $F$, they next generalize its negation $\neg F$ to a hypothesis $H$ such that $H \models \neg F$.

\footnotetext{
${ }^{2}$ This rule is often used to introduce the market basket analysis that detects cross-selling opportunities from the custom behavior.
} 
Example 3 Recall Example 2. Let a ground clausal theory $F_{2}$ be as follows:

$$
\{\text { buy (john, diaper }) \vee \operatorname{buy}(\text { john, beer }), \neg \text { shopping(john,at_night })\} .
$$

Since $F_{2}=B_{2} \cup \neg E_{2}, F_{2}$ is a bridge theory wrt $B_{2}$ and $E_{2}$. We easily have the DNF formula of $\neg F_{2}$ using De Morgan's laws. By translating this DNF formula into CNF with the standard equivalent operations, we get $\neg F_{2}$ as the following clausal theory:

$$
\begin{aligned}
& \left.\neg F_{2}=\{\text { buy (john, diaper }) \supset \text { shopping(john,at_night }\right) \text {, } \\
& \text { buy(john, beer) つ shopping(john,at_night)\}. }
\end{aligned}
$$

The clause (5) is subsumed by the resolvent of two parent clauses (3) and (4) in $H_{2}$. The other clause (6) is also subsumed by the clause (4) in $H_{2}$. Hence, $H_{2} \models \neg F_{2}$ holds, though $\mathrm{H}_{2}$ does not subsume $\neg F_{2}$.

Every IE-based method generalizes the negation of a constructed bridge theory to a hypothesis in its own way. On the one hand, CF-induction (Inoue 2004) generalizes it based on anti-entailment. There are several well-known operators to realize this generalization, such as inverse resolution (Muggleton and Buntine 1988) which applies the inverse of resolution, anti-weakening which adds some clauses, anti-instantiation which replaces ground terms with variables and dropping which drops some literals from a clause. These generalization operators are soundly applied, and can jointly generate any hypothesis $H$ such that $H \models \neg F$. For example, $H_{2}$ is generated from $\neg F_{2}$ in such a way that we first replace the term john in the clause (5) with a variable using anti-instantiation, and next derive the two parent clauses (3) and (4) by applying inverse resolution to the clause (5).

Note here that there are many ways to apply inverse resolution to a clause, because inverse resolution can generate whatever two parent clauses of it. In turn, the other generalization operators are also applicable in many ways. Moreover, any combination of them can be applied as another operator. This fact makes generalization with anti-entailment highly non-deterministic and causes the search space to be very large.

Because of this situation, most IE-based methods (Muggleton 1995; Yamamoto 2003; Ray et al. 2003; Ray and Inoue 2008; Ray 2009; Tamaddoni-Nezhad and Muggleton 2009; Kimber et al. 2009), except for CF-induction, are based on inverse subsumption that generalizes the negation of a bridge theory using anti-subsumption, instead of anti-entailment. Generalization with anti-subsumption has been actively studied in the context of refinement operators (Nienhuys-Cheng and De Wolf 1997; Badea and Stanciu 1999; Bratko 1999; Riguzzi 2005; Tamaddoni-Nezhad and Muggleton 2009). They systematically explore the hypothesis space structured by a bounded subsumption lattice. However, it was not yet clarified how their logical reduction from inverse entailment to inverse subsumption affects the completeness of generalization. For example, though $H_{2}$ can be generated from $\neg F_{2}$ by inverse entailment, inverse subsumption cannot do as $H_{2}$ does not subsume $\neg F_{2}$. For this problem, the following two sections show that given a bridge theory $F$ and a hypothesis $H$ such that $H \models \neg F$, there is a certain clausal theory $F^{*}$ such that $F^{*} \equiv \neg F$ and $H \succeq F^{*}$.

\subsection{Residue and minimal complements}

We define two kinds of clausal theories regarded as the above $F^{*}$. A clausal theory $S$ is irredundant if there is no clause $C \in S$ such that $S-\{C\} \equiv S$; otherwise it is redundant. Note that $S$ becomes redundant if $S$ contains either tautologies or clauses that are properly 
subsumed by others. $\tau(S)$ denotes the clausal theory obtained by removing all the tautologies from $S . \mu(S)$ denotes the clausal theory obtained by removing from $S$ all clauses that are properly subsumed by clauses in $S$. We say $S$ is subsume-minimal if $S=\mu(S)$ holds.

Let $S$ be a ground clausal theory $\left\{C_{1}, C_{2}, \ldots, C_{n}\right\}$ where each clause $C_{i}(1 \leq i \leq n)$ $=l_{i, 1} \vee l_{i, 2} \vee \cdots \vee l_{i, m_{i}}$. The complement of $S$, denoted by $\bar{S}$, is defined as follows:

$$
\bar{S}=\left\{\neg l_{1, k_{1}} \vee \neg l_{2, k_{2}} \vee \cdots \vee \neg l_{n, k_{n}} \quad \begin{array}{c}
1 \leq k_{1} \leq m_{1}, 1 \leq k_{2} \leq m_{2}, \\
\ldots, 1 \leq k_{n} \leq m_{n}
\end{array}\right\} .
$$

In case that $S$ is empty, $\bar{S}$ is defined as the set $\{\perp\}$ where $\perp$ is the empty clause. Note that $\bar{S}$ is a CNF formula such that $\bar{S} \equiv \neg S$. Accordingly, $\tau(\bar{S})$ and $\mu(\bar{S})$ are also CNF formulas logically equivalent to $\neg S$. In the following, we denote $\tau(\bar{S})$ and $\mu(\bar{S})$ as the functions $R(S)$ and $M(S)$, called the residue and minimal complement of $S$, respectively. For sake of simplicity, we often denote $R(R(S))$ and $M(M(S))$ by $R^{2}(S)$ and $M^{2}(S)$, respectively. Note that $R(S) \equiv M(S) \equiv \neg S$ and $R^{2}(S) \equiv M^{2}(S) \equiv S$.

Example 4 Let $S$ be the clausal theory $\{a \vee b, b \vee c, \neg c\}$. Then, $\bar{S}, R(S)$ and $M(S)$ are as below. $M(S)$ is obtained by removing two clauses $\neg a \vee \neg b \vee c$ and $\neg b \vee \neg c \vee c$, which are subsumed by the clause $\neg b \vee c$. Note that $M(S)$ contains a tautology. On the other hand, $R(S)$ is obtained by all of the tautologies in $\bar{S}$, though $R(S)$ contains a redundant clause subsumed by another.

$$
\begin{aligned}
& \bar{S}=\{\neg a \vee \neg b \vee c, \neg a \vee \neg c \vee c, \neg b \vee c, \neg b \vee \neg c \vee c\}, \\
& M(S)=\{\neg a \vee \neg c \vee c, \neg b \vee c\}, \quad R(S)=\{\neg a \vee \neg b \vee c, \neg b \vee c\} .
\end{aligned}
$$

In next two sections, we use the residue and minimal complements as the two kinds of clausal theories representing the above $F^{*}$ such that $H \succeq F^{*}$ holds, and show that inverse subsumption with each of them ensures the completeness for finding hypotheses.

\section{Inverse subsumption with residue complements}

Let $S$ and $T$ be two ground clausal theories such that $S \models T$. Our approach is based on the fact that the logical relation between the two CNF formulas translated from $\neg S$ and $\neg T$ is represented by anti-subsumption. We intend to apply this feature to Relation (1). Since $\neg S$ and $\neg T$ are DNF formulas after applying De Morgan's laws, there are several ways to represent $\neg S$ and $\neg T$ into CNF. In this section, we use the residue complement and consider the logical relation between $R(S)$ and $R(T)$, which is represented primarily by the following theorem ${ }^{3}$.

Theorem 1 (Yamamoto 2003) Let $S$ and $T$ be two clausal theories such that $T$ is ground and both $S$ and $T$ do not include any tautologies. If $S \models T$, there is a finite subset $S^{\prime}$ of ground instances from $S$ such that $R(T) \succeq R\left(S^{\prime}\right)$.

By Theorem 1, the following holds, when $S$ is ground.

\footnotetext{
${ }^{3}$ Theorem 1 and Lemma 1 have been proved in the literature (Yamamoto 2003). We give their alternative proofs in the appendix.
} 
Corollary 1 Let $S$ and $T$ be two ground clausal theories such that $S$ and $T$ do not include any tautologies. If $S \models T$, then $R(T) \succeq R(S)$.

We first recall the following lemma ${ }^{4}$ to prove Corollary 1.

Lemma 1 (Yamamoto 2003) For ground clausal theories $S$ and $T$ that do not include tautologies, $T \subseteq S$ implies $R(T) \succeq R(S)$.

Using Lemma 1 and Theorem 1, Corollary 1 is proved as follows:

Proof of Corollary 1 By Theorem 1, there is a ground theory $S^{\prime}$ such that $S^{\prime} \subseteq S$ such that $R(T) \succeq R\left(S^{\prime}\right)$. By Lemma $1, R\left(S^{\prime}\right) \succeq R(S)$ holds. Hence, $R(T) \succeq R(S)$ holds.

We apply Corollary 1 to the logical relation $F \models \neg H$ where $F$ is a bridge theory and $H$ is a ground hypothesis. We represent $\neg H$ using the residue complement $R(H)$. Suppose that $F$ does not include any tautologies. Then, by Corollary $1, R^{2}(H) \succeq R(F)$ holds. In other words, $R^{2}(H)$, which is logically equivalent to $H$, can be obtained from $R(F)$ using anti-subsumption.

Theorem 2 Let $F$ be a bridge theory such that $F$ does not include tautologies, and $H$ be a hypothesis such that $F \models \neg H$. Then, there is a hypothesis $H^{*}$ such that $H^{*} \equiv H$ and $H^{*} \succeq R(F)$.

Proof of Theorem 2 By Herbrand's theorem, ${ }^{5}$ there is a ground clausal theory $H_{g}$ such that $H \succeq H_{g}$ and $F \models \neg H_{g}$. Since $\neg H_{g} \equiv R\left(H_{g}\right)$ holds, $R^{2}\left(H_{g}\right) \succeq R(F)$ holds by Corollary 1 . Assume the clausal theory $H^{*}=H \cup R^{2}\left(H_{g}\right)$. Since $H \succeq H_{g}$ and $H_{g} \equiv R^{2}\left(H_{g}\right), H \models$ $R^{2}\left(H_{g}\right)$ holds. Accordingly, $H \models H^{*}$ holds. Hence, $H^{*} \equiv H$ holds. Since $R^{2}\left(H_{g}\right) \succeq R(F)$ and $H^{*} \supseteq R^{2}\left(H_{g}\right), H^{*} \succeq R(F)$ holds.

Example 5 Let $B_{3}, E_{3}$ and $H_{3}$ be a background theory, examples and a target hypothesis as follows:

$$
\begin{aligned}
& B_{3}=\{p \supset q\}, \quad E_{3}=\{p \supset r\}, \\
& H_{3}=\{q \supset r\} .
\end{aligned}
$$

Suppose the clausal theory $F_{3}=\{p \supset q, p, \neg r\}$. Since $\neg E_{3}=p \wedge \neg r, F_{3}$ corresponds to $B_{3} \wedge \neg E_{3}$. Then, $F_{3}$ is a bridge theory wrt $B_{3}$ and $E_{3}$ such that $F_{3} \models \neg H_{3}$. The residue complement $R\left(F_{3}\right)$ is $\{\neg q \vee p \vee r\}$. We notice that $R\left(F_{3}\right)$ is subsumed by $H_{3}$. Hence, the hypothesis $H_{3}$ can be obtained from $R\left(F_{3}\right)$ using anti-subsumption.

Theorem 2 means that for every hypothesis $H$, its equivalent hypothesis $H^{*}$ can be derived from the residue complement $R(F)$ using anti-subsumption. In this sense, inverse subsumption with residue complements ensures the completeness of generalization.

\footnotetext{
${ }^{4}$ In case that $T$ is empty, $\bar{T}=\{\perp\}$ holds. Since $R(T)=\tau(\bar{T}), R(T)$ contains the empty clause $\perp$ which subsumes any clause. Hence, for any clausal theory $S, R(T) \succeq R(S)$ holds.

${ }^{5} \mathrm{~A}$ set of clauses $\Sigma$ is unsatisfiable if and only if a finite set of ground instances of clauses of $\Sigma$ is unsatisfiable (Chang and Lee 1973).
} 
However, every target hypothesis itself is not necessarily obtained from the residue complement by anti-subsumption. The below example describes such a case.

Example 6 Let $B_{4}, E_{4}$ and $H_{4}$ be a background theory, examples and a target hypothesis as follows:

$$
\begin{aligned}
& B_{4}=\{p(a)\}, \quad E_{4}=\{p(f(f(a))\}, \\
& H_{4}=\{p(a) \supset p(f(a)), p(f(a)) \supset p(f(f(a)))\} .
\end{aligned}
$$

Let $F_{4}$ be the clausal theory $\{p(a), \neg p(f(f(a)))\}$. Since $F_{4}$ corresponds to $B_{4} \wedge \neg E_{4}, F_{4}$ is a bridge theory wrt $B_{4}$ and $E_{4}$ such that $F_{4} \models \neg H_{4} . R\left(F_{4}\right)$ is $\{p(a) \supset p(f(f(a)))\}$. Then we notice that $R\left(F_{4}\right)$ is not subsumed by $H_{4}$. Indeed, $R\left(F_{4}\right)$ is the resolvent of two clauses in $H_{4}$. Hence, we need to apply an inverse resolution operator to $R\left(F_{4}\right)$ for obtaining the target hypothesis $H_{4}$. Note that $R\left(H_{4}\right)$ and $R^{2}\left(H_{4}\right)$ are as follows:

$$
\begin{aligned}
& R\left(H_{4}\right)=\{p(a) \vee p(f(a)), p(a) \vee \neg p(f(f(a))), \neg p(f(a)) \vee \neg p(f(f(a)))\}, \\
& R^{2}\left(H_{4}\right)=\{\neg p(a) \vee p(f(a)), \neg p(a) \vee p(f(f(a))), \neg p(f(a)) \vee p(f(f(a))), \\
& \neg p(a) \vee p(f(f(a))) \vee p(f(a)), \neg p(a) \vee p(f(f(a)) \vee \neg p(f(a))\} .
\end{aligned}
$$

We notice that $R^{2}\left(H_{4}\right)$ contains the unique clause $\neg p(a) \vee p(f(f(a)))$ in $R\left(F_{4}\right)$. Hence, $R^{2}\left(H_{4}\right)$ subsumes $R\left(F_{4}\right)$. Since $R^{2}\left(H_{4}\right) \equiv H_{4}$, Theorem 2 holds by regarding the equivalent hypothesis $H_{4}^{*}$ as $R^{2}\left(H_{4}\right)$.

The problem described in the above example is caused by the fact that $R^{2}(H)=H$ cannot necessarily hold. Indeed, the key idea in Theorem 2 lies in the logical relation $R^{2}(H) \succeq R(F)$. If $R^{2}(H)=H$ should not hold, $H$ cannot be obtained from $R(F)$ using anti-subsumption. We thus need another CNF formula $F(H)$ for representing the negation of a hypothesis $H$ such that $F(F(H))=H$.

\section{Inverse subsumption with minimal complements}

\subsection{Properties of minimal complements}

We here investigate minimal complements. Firstly, the following theorem holds.

Theorem 3 Let $S$ be a ground clausal theory. Then, $M^{2}(S)=\mu(S)$ holds.

Proof The proof of Theorem 3 is given in the appendix.

This theorem can be regarded as a fixpoint theorem on the function $M$ computing the minimal complement of $\mu(S)$. Unlike residue complements, $M^{2}(S)$ corresponds to $S$ itself in case that $S$ is subsume-minimal. Thus, minimal complements may not cause the problem of residue complements that they cannot necessarily obtain a target hypothesis using antisubsumption, as described in Sect. 3. 
Example 7 We recall Example 4. Then, $\bar{S}, R(S), R^{2}(S), M(S)$ and $M^{2}(S)$ are as follows. In fact, $M^{2}(S)=S$ holds, whereas $R^{2}(S)$ does not.

$$
\begin{aligned}
& \bar{S}=\{\neg a \vee \neg b \vee c, \neg a \vee \neg c \vee c, \neg b \vee c, \neg b \vee \neg c \vee c\}, \\
& R(S)=\{\neg a \vee \neg b \vee c, \neg b \vee c\}, \quad R^{2}(S)=\{a \vee b, \neg c \vee a, b, \neg c \vee b, \neg c\}, \\
& M(S)=\{\neg a \vee \neg c \vee c, \neg b \vee c\}, \quad M^{2}(S)=\{a \vee b, b \vee c, \neg c\} .
\end{aligned}
$$

On the other hand, unlike residue complements, the logical relation $M(T) \succeq M(S)$ does not necessarily hold whenever $S \models T$ holds for ground clausal theories $S$ and $T$.

Example 8 We recall Example 6. $M\left(H_{4}\right)$ is as follows:

$\{p(a) \vee p(f(a)), p(a) \vee \neg p(f(f(a))), \neg p(f(a)) \vee \neg p(f(f(a))), \neg p(f(a)) \vee p(f(a))\}$.

Suppose the same bridge theory $F_{4}=\{p(a), \neg p(f(f(a)))\}$. Then, $F_{4} \models M\left(H_{4}\right)$ holds. But, $M^{2}\left(H_{4}\right) \succeq M\left(F_{4}\right)$ does not hold. Because $M^{2}\left(H_{4}\right)$ corresponds to $H_{4}$ by Theorem 3 , and $H_{4}$ does not subsume $M\left(F_{4}\right)=\{\neg p(a) \vee p(f(f(a)))\}$.

This is because minimal complements can include tautologies that residue complements never have. Indeed, Corollary 1, which shows the logical relation between $R(T)$ and $R(S)$, does not allow any tautologies to be included in $S$ and $T$. We then extend Corollary 1 so as to deal with tautologies as follows:

Theorem 4 Let $S$ and $T$ be ground clausal theories such that $S \models T$ and for every tautology $D \in T$, there is a clause $C \in S$ such that $C \succeq D$. Then,

$$
\tau(M(T)) \succeq \tau(M(S)) .
$$

Proof The proof of Theorem 4 is given in the Appendix B.

Example 9 Recall Example 6. We have $F_{4} \models M\left(H_{4}\right)$, but $M\left(H_{4}\right)$ contains one tautology: $\neg p(f(a)) \vee p(f(a))$, which is not subsumed by any clause in $F_{4}$. Suppose that this tautology is added to $F_{4}$. We denote by $F_{4}^{\prime}$ the added clausal theory. Since $F_{4}^{\prime}=$ $\{p(a), \neg p(f(f(a))), \neg p(f(a)) \vee p(f(a))\}, \tau\left(M\left(F_{4}^{\prime}\right)\right)$ is as follows:

$$
\begin{gathered}
\{\neg p(a) \vee p(f(f(a))) \vee \neg p(f(a)) \\
\hdashline \neg p(a) \vee p(f(f(a)) \vee p(f(a))\}
\end{gathered}
$$

We then notice that $H_{4}$ subsumes $\tau\left(M\left(F_{4}^{\prime}\right)\right.$ ) (See the dotted surrounding parts). This subsumption relation can be derived using Theorem 4 . Since $F_{4}^{\prime} \equiv F_{4}$ and $F_{4} \models M\left(H_{4}\right)$, it holds that $F_{4}^{\prime} \models M\left(H_{4}\right)$. Since the tautology in $M\left(H_{4}\right)$ is also contained in $F_{4}^{\prime}$, we can use Theorem 4 , and then have $\tau\left(M^{2}\left(H_{4}\right)\right) \succeq \tau\left(M\left(F_{4}^{\prime}\right)\right)$. By Theorem 3, $\tau\left(M^{2}\left(H_{4}\right)\right)=\tau\left(\mu\left(H_{4}\right)\right)$ holds. Since $H_{4}$ is subsume-minimal and it does not contain any tautologies, it holds that $\tau\left(\mu\left(H_{4}\right)\right)=H_{4}$. Hence, we obtain $H_{4} \succeq \tau\left(M\left(F_{4}^{\prime}\right)\right)$. 


\subsection{Generalization with minimal complements}

Theorems 3 and 4 enable us to construct an alternative generalization procedure using minimal complements. To describe the hypotheses that can be found by this, we first introduce the following language bias, called an induction field:

Definition 4 (Induction field) An induction field, denoted by $\mathcal{I}_{H}=\langle\mathbf{L}\rangle$, where $\mathbf{L}$ is a finite set of literals to appear in ground hypotheses. A ground hypothesis $H_{g}$ belongs to $\mathcal{I}_{H}$ if every literal in $H_{g}$ is included in $\mathbf{L}$. Given an induction field $\mathcal{I}_{H}=\langle\mathbf{L}\rangle$, $\operatorname{Taut}\left(\mathcal{I}_{H}\right)$ is defined as the set of tautologies $\{\neg A \vee A \mid A \in \mathbf{L}$ and $\neg A \in \mathbf{L}\}$.

We next define the target hypotheses using the notion of an induction field $\mathcal{I}_{H}$, together with a bridge theory $F$ as follows:

Definition 5 (Hypothesis wrt $\mathcal{I}_{H}$ and $F$ ) Let $H$ be a hypothesis. $H$ is a hypothesis wrt $\mathcal{I}_{H}$ and $F$ if there is a ground hypothesis $H_{g}$ such that $H_{g}$ consists of instances from $H$, $F \models \neg H_{g}$ and $H_{g}$ belongs to $\mathcal{I}_{H}$.

Now, the generalization procedure based on inverse subsumption with minimal complements is as follows:

Definition 6 Let $B, E$ and $\mathcal{I}_{H}=\langle\mathbf{L}\rangle$ be a background theory, examples and an induction field, respectively. Let $F$ be a bridge theory wrt $B$ and $E$. A clausal theory $H$ is derived by inverse subsumption with minimal complements from $F$ wrt $\mathcal{I}_{H}$ if $H$ is constructed as follows.

Step 1. Compute $\operatorname{Taut}\left(\mathcal{I}_{H}\right)$;

Step 2. Compute $\tau\left(M\left(F \cup \operatorname{Taut}\left(\mathcal{I}_{H}\right)\right)\right)$;

Step 3. Construct a clausal theory $H$ satisfying the condition:

$$
H \succeq \tau\left(M\left(F \cup \operatorname{Taut}\left(\mathcal{I}_{H}\right)\right)\right) .
$$

Inverse subsumption with minimal complements ensures the completeness for finding hypotheses wrt $\mathcal{I}_{H}$ and $F$, by way of (7).

Main Theorem Let $B, E$ and $\mathcal{I}_{H}$ be a background theory, examples and an induction field, respectively. Let $F$ be a bridge theory wrt $B$ and $E$. For every hypothesis $H$ wrt $\mathcal{I}_{H}$ and $F$, $H$ is derived by inverse subsumption with minimal complements from $F \operatorname{wrt} \mathcal{I}_{H}$.

Proof of Main Theorem It is sufficient to prove the following lemma.

Lemma 2 Let $B, E$ and $\mathcal{I}_{H}$ be a background theory, examples and an induction field, respectively. Let $F$ be a bridge theory wrt $B$ and $E$. For every hypothesis $H$ wrt $\mathcal{I}_{H}$ and $F$, $H$ satisfies the following condition:

$$
H \succeq \tau\left(M\left(F \cup \operatorname{Taut}\left(\mathcal{I}_{H}\right)\right)\right) .
$$

Proof of Lemma 2 Since $H$ is a hypothesis wrt $\mathcal{I}_{H}$ and $F$, there is a ground hypothesis $H_{g}$ such that $H \succeq H_{g}, F \models \neg H_{g}$ and $H_{g}$ belongs to $\mathcal{I}_{H}$. Since $\neg H_{g} \equiv M\left(H_{g}\right), F \models M\left(H_{g}\right)$ 
holds. Accordingly, $F \cup \operatorname{Taut}\left(\mathcal{I}_{H}\right) \models M\left(H_{g}\right)$ holds. Since $H_{g}$ belongs to $\mathcal{I}_{H}$, every literal in $H_{g}$ is included in $\mathcal{I}_{H}$. Then, for every tautological clause $D \in M\left(H_{g}\right)$, there is a clause $C \in \operatorname{Taut}\left(\mathcal{I}_{H}\right)$ such that $C \succeq D$. By Theorem 4, $\tau\left(M^{2}\left(H_{g}\right)\right) \succeq \tau\left(M\left(F \cup \operatorname{Taut}\left(\mathcal{I}_{H}\right)\right)\right)$ holds. Since $\mu\left(H_{g}\right)=M^{2}\left(H_{g}\right)$ by Theorem 3, $\tau\left(\mu\left(H_{g}\right)\right) \succeq \tau\left(M\left(F \cup \operatorname{Taut}\left(\mathcal{I}_{H}\right)\right)\right)$ holds. Since $H_{g} \supseteq \tau\left(\mu\left(H_{g}\right)\right), H_{g} \succeq \tau\left(\mu\left(H_{g}\right)\right)$ holds. Hence, $H \succeq \tau\left(M\left(F \cup \operatorname{Taut}\left(\mathcal{I}_{H}\right)\right)\right)$ holds.

\subsection{Examples}

We show how a target hypothesis is derived by inverse subsumption with minimal complements using the below examples.

Example 10 Recall Example 6 that could not be solved using residue complements. Let an induction field $\mathcal{I}_{\mathrm{H}_{4}}$ be as follows:

$$
\mathcal{I}_{H_{4}}=\langle\{\neg p(a), p(f(a)), \neg p(f(a)), p(f(f(a)))\}\rangle .
$$

$H_{4}$ belongs to $\mathcal{I}_{H_{4}}$ and $H_{4} \models \neg F_{4}$ holds. Then, $H_{4}$ is a hypothesis wrt $\mathcal{I}_{H_{4}}$ and $F_{4}$. $\operatorname{Taut}\left(\mathcal{I}_{H_{4}}\right)$ is the set $\{p(f(a)) \vee \neg p(f(a))\}$. Note that $F_{4} \cup \operatorname{Taut}\left(\mathcal{I}_{H_{4}}\right)$ corresponds to $F_{4}^{\prime}$ in Example 9. Then, $H_{4}$ subsumes $\tau\left(M\left(F_{4} \cup \operatorname{Taut}\left(\mathcal{I}_{H_{4}}\right)\right)\right)$ as shown in Example 9 . Hence, $H_{4}$ is derivable by inverse subsumption with minimal complements.

Example 11 Recall $H_{2}$ and $F_{2}$ in Example 3. $R\left(F_{2}\right)$ corresponds to $\neg F_{2}$ consisting of two clauses (5) and (6). Then, $H_{2}$ does not subsume $R\left(F_{2}\right)$, and cannot be generated from $R\left(F_{2}\right)$ by inverse subsumption. In contrast, it is derivable by inverse subsumption with minimal complement. Let an induction field $\mathcal{I}_{H_{2}}$ be as follows:

$\langle\{\neg$ buy(john, diaper), buy(john, beer), $\neg$ buy(john, beer), shopping(john,at_night)\}〉.

Consider the following ground hypothesis $\mathrm{H}_{g_{2}}$ consisting of instances from $\mathrm{H}_{2}$ :

$$
\begin{aligned}
H_{g_{2}}=\{\text { buy }(\text { john }, \text { diaper }) & \supset \text { buy }(\text { john }, \text { beer }), \\
\text { buy }(\text { john, beer }) & \supset \operatorname{shopping~}(\text { john, at_night })\} .
\end{aligned}
$$

$H_{g_{2}}$ belongs to $\mathcal{I}_{H_{2}}$ and $F_{2} \models \neg H_{g_{2}}$ holds. Then, $H_{2}$ is a hypothesis wrt $\mathcal{I}_{H_{2}}$ and $F_{2}$. $\operatorname{Taut}\left(\mathcal{I}_{\mathrm{H}_{2}}\right)$ contains one tautology: buy(john, beer $) \vee \neg$ buy(john, beer). After adding this tautology to $F_{2}$, we compute $\tau\left(M\left(F_{2} \cup \operatorname{Taut}\left(\mathcal{I}_{\mathrm{H}_{2}}\right)\right)\right)$ represented as follows.

$$
\left\{\begin{array}{c:c}
\neg \text { buy }(\text { john, diaper }) \vee \text { buy }(\text { john, beer }) \vee \operatorname{shopping}(j o h n, \text { at_night }), \\
\hdashline \neg \text { buy }(\text { john, beer }) \vee \operatorname{shopping}(\text { john, at_night }) \\
\end{array}\right\} .
$$

Then, $H_{2}$ indeed subsumes $\tau\left(M\left(F_{2} \cup \operatorname{Taut}\left(\mathcal{I}_{H_{2}}\right)\right)\right)$ (See the dotted surrounding parts). Hence, $H_{2}$ can be generated by inverse subsumption with this minimal complement.

Example 12 We next consider the following example on pathway completion:

$$
\begin{aligned}
& B_{5}=\{\operatorname{arc}(a, b), \operatorname{arc}(X, Y) \wedge \operatorname{path}(Y, Z) \supset \operatorname{path}(X, Z)\}, \quad E_{5}=\{\operatorname{path}(a, c)\}, \\
& \mathcal{I}_{H_{5}}=\{\operatorname{arc}(b, c), \neg \operatorname{arc}(b, c), \operatorname{path}(b, c), \neg \operatorname{path}(b, c)\}, \\
& H_{5}=\{\operatorname{arc}(b, c), \operatorname{arc}(X, Y) \supset \operatorname{path}(X, Y)\} .
\end{aligned}
$$


Note that $\operatorname{arc}(X, Y)$ (resp. path $(X, Y))$ means there is an arc (resp. a path) from a node $X$ to a node $Y . B_{5}$ contains one fact that there is an arc from $a$ to $b$ and one rule that, if there is an arc from $X$ to $Y$ and a path from $Y$ to $Z$, then there is a path from $X$ to $Y$. However, only $B_{5}$ cannot logically explain $E_{5}$ that there is a path from $a$ to $c$. One possible cause is that one arc from $b$ to $c$ and another rule defining the concept of pathways are missing in the background theory. Then, we seek for the hypothesis $H_{5}$ that completes these missing fact and rule. To complete $H_{5}$, both abduction and induction must involve, but most current ILP systems cannot compute it. This advanced inference has a possibility to be effectively applied when we need to complete both facts and rules that are missing in a prior background theory. In fact, there is a recent work to use it for finding master reactions from incomplete biochemical networks in systems biology (Yamamoto et al. 2009b).

Let $F_{5}$ be the clausal theory $\{\operatorname{arc}(a, b), \operatorname{arc}(a, b) \wedge \operatorname{path}(b, c) \supset \operatorname{path}(a, c), \neg \operatorname{path}(a, c)\}$. Since $F_{5}$ is the set of ground instances from $B_{5} \wedge \neg E_{5}, F_{5}$ is a bridge theory wrt $B_{5}$ and $E_{5}$. Since there is a ground hypothesis $H_{g_{5}}=\{\operatorname{arc}(b, c), \operatorname{arc}(b, c) \supset \operatorname{path}(b, c)\}$ such that $H_{g_{5}}$ consists of instances from $H_{5}, F_{5} \models \neg H_{g_{5}}$ and $H_{g_{5}}$ belongs to $\mathcal{I}_{H_{5}}$, $H_{5}$ is a hypothesis wrt $\mathcal{I}_{H_{5}}$ and $F_{5}$. Then, $H_{5}$ could be derived by inverse subsumption with minimal complements. We first compute $\operatorname{Taut}\left(\mathcal{I}_{H_{5}}\right)$. Then, $\operatorname{Taut}\left(\mathcal{I}_{H_{5}}\right)$ is the set $\{\neg \operatorname{arc}(b, c) \vee \operatorname{arc}(b, c), \neg$ path $(b, c) \vee \operatorname{path}(b, c)\}$. After adding $\operatorname{Taut}\left(\mathcal{I}_{H_{5}}\right)$ to $F_{5}$, we compute $\tau\left(M\left(F \cup \operatorname{Taut}\left(\mathcal{I}_{\mathrm{H}_{5}}\right)\right)\right)$ represented as follows.

$$
\begin{aligned}
& \{\neg \operatorname{arc}(a, b) \vee \operatorname{path}(b, c) \vee \operatorname{arc}(b, c) \vee \operatorname{path}(a, c), \\
& \neg \operatorname{arc}(a, b) \vee \neg \operatorname{arc}(b, c) \vee \operatorname{path}(b, c) \vee \operatorname{path}(a, c)\} .
\end{aligned}
$$

We then notice that $H_{5}$ subsumes $\tau\left(M\left(F \cup \operatorname{Taut}\left(\mathcal{I}_{H_{5}}\right)\right)\right)$ (See the dotted surrounding parts). Therefore, $H_{5}$ can be derived by inverse subsumption with minimal complements.

In contrast, Since $R\left(F_{5}\right)$ is $\{\neg \operatorname{arc}(a, b) \vee \operatorname{path}(b, c) \vee \operatorname{path}(a, c)\}, H_{5}$ does not subsume the residue $R\left(F_{5}\right)$. Hence, $H_{5}$ cannot be obtained from the residue complement, whereas the minimal complement can do with inverse subsumption.

Example 13 We lastly consider a biological example to find cellular regulations.

$$
\begin{aligned}
& B_{6}=\{\text { glucose_ext } \supset \text { induced }(\text { hxt }) \vee \operatorname{active}(\operatorname{snf} 3)\}, \\
& E_{6}=\{\text { glucose_ext } \supset \text { glycolysis_on }\}, \\
& H_{6}=\{\text { active }(\text { snf } 3) \supset \text { induced }(\text { hxt }), \text { induced }(h x t) \supset \text { glycolysis_on }\} .
\end{aligned}
$$

Most eukaryotic cells, including yeasts and humans, can sense the availability of carbon sources in their surroundings and, in the presence of their favorite sugar (often glucose), they transport glucose into the cell and use it through the glycolysis pathway to produce energy (Westergaard et al. 2006). The example $E_{6}$ describes this causality between glucose and glycolysis. Now, we know that if glucose is available, the hexose transporter Hxt can be induced or the sensing protein $\mathrm{Snf} 3$ can be active. This prior background theory $B_{6}$ cannot logically explain $E_{6}$, and thus there are some missing links between $B_{6}$ and $E_{6}$. In recent work (Westergaard et al. 2006), it has been made known that a signal triggered by Snf3 leads to induce Hxt, and then glucose can be moved into the cell via the transporter Hxt. Then, $H_{6}$, which describes these cellular regulations, is a considerable hypothesis. However, it is not straightforward for most current IE-based methods to generate the target hypothesis. In the following, we show how our proposal can solve this example. Let a bridge theory $F_{6}$ be $B_{6} \wedge \neg E_{6}$. We give the induction field $\mathcal{I}_{H_{6}}$ as follows:

$$
\langle\{\neg \operatorname{active}(\operatorname{snf} 3), \text { induced }(h x t), \neg \text { induced }(h x t), \text { glycolysis_on }\}\rangle .
$$


Since $H_{6}$ belongs to $\mathcal{I}_{H_{6}}$ and $F_{6} \models \neg H_{6}$ holds, $H_{6}$ is a hypothesis wrt $\mathcal{I}_{H_{6}}$ and $F_{6}$. $\operatorname{Taut}\left(\mathcal{I}_{H_{6}}\right)$ contains one tautology: induced $(h x t) \vee \neg$ induced $(h x t)$. After adding the tautology to $F_{6}$, we compute $\tau\left(M\left(F_{6} \cup \operatorname{Taut}\left(\mathcal{I}_{H_{6}}\right)\right)\right)$ as follows:

$$
\begin{gathered}
\{\neg \text { glucose_ext } \vee \neg \text { active }(\text { snf } 3) \vee \text { induced }(h x t) \vee \text { glycolysis_on, } \\
\quad \neg \text { glucose_ext } \vee \neg \text { induced }(h x t) \vee \text { glycolysis_on }\} .
\end{gathered}
$$

$H_{6}$ subsumes $\tau\left(M\left(F_{6} \cup \operatorname{Taut}\left(\mathcal{I}_{H_{6}}\right)\right)\right)$ (See the dotted surrounding parts). Then, $H_{6}$ is derivable by inverse subsumption with minimal complements. Note that the residue complement $R\left(F_{6}\right)$ is as follows:

$$
\begin{aligned}
& \{\neg \text { glucose_ext } \vee \neg \text { active }(\text { snf } 3) \vee \text { glycolysis_on, } \\
& \quad \neg \text { glucose_ext } \vee \neg \text { induced }(h x t) \vee \text { glycolysis_on }\} .
\end{aligned}
$$

Then, $H_{6}$ does not subsume $R\left(F_{6}\right)$, and then cannot be generated by inverse subsumption with residue complements.

\section{Further topics and related work}

\subsection{The commutative property of residue and minimal complements}

We have proposed two approaches with residue and minimal complements for inverse subsumption. The derivable hypotheses in two approaches are characterized as Theorem 2 and Main Theorem, respectively. In this section, we clarify some commonness between these approaches and investigate what aspect causes their crucial difference.

Lemma 3 Let $S$ be a clausal theory. Then, $\tau(\mu(S))=\mu(\tau(S))$.

Proof of Lemma 3 We show that, for any clause $C \in S, C \notin \mu(\tau(S))$ if and only if $C \notin$ $\tau(\mu(S))$. $(\Rightarrow)$ Suppose $C \notin \mu(\tau(S))$. There is a clause $D \in \tau(S)$ such that $D \succeq C$. Since $D \in S$ holds, we have $C \notin \mu(S)$. Then, $C \notin \tau(\mu(S))$ holds. $(\Leftarrow)$ Suppose $C \notin \tau(\mu(S))$. $C$ is a tautology or there is a clause $D \in S$ such that $D \succeq C$. If $C$ is a tautology, $C \notin \mu(\tau(S))$ holds. In the other case, since $D \succeq C$ and $C$ is not a tautology, $D$ is also not, that is, $D \in \tau(S)$ holds. Then, $C \notin \mu(\tau(S))$ holds.

By Lemma 3, $\tau(M(S))=\mu(R(S))$ holds, since $\tau(M(S))=\tau(\mu(\bar{S}))$ and $\mu(R(S))=$ $\mu(\tau(\bar{S}))$. Hence, residue and minimal complements satisfy the commutative property. Using this property, we obtain a new variation by replacing $\tau(M(S))$ with $\mu(R(S))$ in Lemma 2 as follows:

Corollary 2 Let $H$ be a hypothesis wrt $\mathcal{I}_{H}$ and $F$. Then, $H \succeq R\left(F \cup \operatorname{Taut}\left(\mathcal{I}_{H}\right)\right)$.

Proof of Corollary 2 By Lemma 2, $H \succeq \tau\left(M\left(F \cup \operatorname{Taut}\left(\mathcal{I}_{H}\right)\right)\right)$ holds. Since $\mu(R(S))=$ $\tau(M(S)), H \succeq \mu\left(R\left(F \cup \operatorname{Taut}\left(\mathcal{I}_{H}\right)\right)\right)$, and thus $H \succeq R\left(F \cup \operatorname{Taut}\left(\mathcal{I}_{H}\right)\right)$ holds.

Every hypothesis wrt $\mathcal{I}_{H}$ and $F$ is also derivable by inverse subsumption with residue complements by adding $\operatorname{Taut}\left(\mathcal{I}_{H}\right)$ to the original bridge theory. In contrast, even if the tautologies are not added, inverse subsumption with minimal complements ensures the equivalent completeness in the case of residue complements. 
Corollary 3 Let $F$ be a bridge theory without tautologies, and $H$ be a hypothesis such that $F \models \neg H$. Then, there is a hypothesis $H^{*}$ such that $H^{*} \equiv H$ and $H^{*} \succeq \tau(M(F))$.

Proof of Corollary 3 By Theorem 2, there is a hypothesis $H^{*}$ such that $H^{*} \equiv H$ and $H^{*} \succeq R(F)$. Since $R(F) \succeq \mu(R(F)), H^{*} \succeq \mu(R(F))$ holds. Since $\mu(R(F))=\tau(M(F))$, we get $H^{*} \succeq \tau(M(F))$.

The completeness of inverse subsumption with either residue or minimal complements is varied by whether adding tautologies or not. In the case of adding tautologies, both approaches can derive every hypothesis $H$ wrt $\mathcal{I}_{H}$ and $F$. In the other case, they can derive its equivalent hypothesis $H^{*}$, which can be characterized as follows:

Definition 7 (Maximal Hypothesis) Let $H$ be a hypothesis. $H$ is a maximal hypothesis if, for each consequence $C$ of $H$, there is a clause $D$ in $H$ such that $D \succeq C$.

Example 14 Let $H_{7}$ and $H_{8}$ be two hypotheses as follows:

$$
H_{7}=\{p(X) \supset p(f(X))\}, \quad H_{8}=\{p(a), p(X) \supset q(X)\} .
$$

$H_{7}$ is not a maximal hypothesis since a consequence $p(X) \supset p(f(f(X)))$ of $H_{7}$ is not contained in $H_{7} . H_{8}$ is also not, since the consequence $q(a)$ is not in $H_{8}$. In contrast, $H_{8} \cup$ $\{q(a)\}$ is a maximal hypothesis. Note that, like non-recursive rules, any hypothesis where no derivations exist is a maximal hypothesis.

Maximal hypotheses are derivable by inverse subsumption even if tautologies are not added.

Corollary 4 Let $H$ be a maximal hypothesis wrt $\mathcal{I}_{H}$ and $F$. Then, $H \succeq R(F)$.

Proof of Corollary 4 There is a maximal hypothesis $H_{g}$ such that $H_{g}$ consists of ground instances from $H$ and $F \models \neg H_{g}$. Since $\neg H_{g} \equiv R\left(H_{g}\right), F \models R\left(H_{g}\right)$ holds. By Corollary 1 , we have $R^{2}\left(H_{g}\right) \succeq R(F)$. Since $H_{g}$ is a maximal hypothesis, for every consequence $C$ of $H_{g}$, there is a clause $D \in H_{g}$ such that $D \succeq C$. Since $H_{g} \equiv R^{2}\left(H_{g}\right)$, every clause in $R^{2}\left(H_{g}\right)$ is regarded as a consequence of $H_{g}$. Then, $H_{g} \succeq R^{2}\left(H_{g}\right)$ holds. Hence, we get $H \succeq R(F)$ because of $H \succeq H_{g}$ and $R^{2}\left(H_{g}\right) \succeq R(F)$.

Corollary 5 Let $H$ be a maximal hypothesis wrt $\mathcal{I}_{H}$ and $F$. Then, $H \succeq \tau(M(F))$.

Proof of Corollary 5 By Corollary 4, $H \succeq R(F)$ holds. Since $\mu(R(F))=\tau(M(F))$ and $R(F) \succeq \mu(R(F))$, we get $H \succeq \tau(M(F))$.

\subsection{Embedding inverse subsumption to IE-based methods}

The results in the paper can be applied to previously proposed IE-based methods. Firstly, we review those methods in brief.

Progol (Muggleton 1995; Tamaddoni-Nezhad and Muggleton 2009), one of the state-ofthe-art ILP systems in Horn clause learning, uses the technique of Bottom Generalization. Its bridge theory $F$ corresponds to the conjunction of ground literals each of which is derived from $B$ and $\neg E$. After constructing $\neg F$ called the bottom clause $\perp(B, E)$, Progol generalizes it with anti-subsumption, instead of anti-entailment. 
HAIL (Ray et al. 2003; Ray and Inoue 2008) constructs so-called Kernel Sets to overcome some limitation on Bottom Generalization. Each ground clause $C_{i}$ in a Kernel Set $K S=\left\{C_{1}, \ldots, C_{n}\right\}$ is given by the form of $B_{1}^{i} \wedge \cdots \wedge B_{m_{i}}^{i} \supset A^{i}$, where $B \cup\left\{A^{1}, \ldots, A^{n}\right\} \models$ $E$ and $B \models\left\{B_{1}^{1}, \ldots, B_{m_{n}}^{n}\right\}$. After constructing a Kernel Set, HAIL also generalizes it using anti-subsumption like Progol. A Kernel Set $K S$ is regarded as the negation of a certain bridge theory $F$. In other words, HAIL directly constructs the negation of $F$ by separately computing head and body literals of each clause in the negation.

Example 15 We recall Example 6. The bottom clause $\perp\left(B_{4}, E_{4}\right)=p(a) \supset p(f(f(a)))$ and the Kernel set $K S$ only contains the bottom clause. Then, $H_{4}$ does not subsume neither $\perp\left(B_{4}, E_{4}\right)$ nor $K S$. Hence, both Progol and HAIL cannot solve this example.

We remark there is an extension called X-HAIL (Ray 2009) which allows the body in a Kernel Set $K S$ to contain such literals that are derived by $B$ with the head literals of $K S$. There is a recent work to extend Kernel Sets into so-called Connection Theories with an iterative procedure, called Imparo (Kimber et al. 2009). Note that X-HAIL and Imparo can solve Example 6.

The residue procedure (Yamamoto 2003), which has been firstly proposed to find hypotheses in full clausal theories, constructs a bridge theory $F$ consisting of ground instances from $B \wedge \overline{E \sigma}$, where $\sigma$ is a ground substitution to skolemize $E$. It then computes the residue complement $R(F)$, and generalizes it with anti-subsumption. In contrast, CF-induction (Inoue 2004) is sound and complete for finding hypotheses in full clausal theories. It constructs a bridge theory $F$ consisting of ground instances from so-called characteristic clauses of $B \wedge \overline{E \sigma}$. Each characteristic clause is a subsume-minimal consequence of $B \wedge \overline{E \sigma}$ that satisfies a given language bias. Then CF-induction translates the DNF formula $\neg F$ into a CNF formula and generalizes it with anti-entailment.

Every method in the above, except for CF-induction, is based on inverse subsumption. Hence, it reduces anti-entailment to anti-subsumption. Based on our result, we investigate the completeness of generalization in those IS-based methods.

Definition 8 (Completeness in generalization) Suppose an IS-based method $\Gamma$. $\Gamma$ is (resp. partially) complete in generalization if for each bridge theory $F_{\Gamma}$ of $\Gamma$ and each induction field $\mathcal{I}_{H}, \Gamma$ derives any (resp. maximal) hypothesis wrt $\mathcal{I}_{H}$ and $F_{\Gamma}$.

For simplicity, we denote by $p, h, x, i$ and $r$ Progol, HAIL, X-HAIL, Imparo and Residue procedure, respectively. Note that HAIL (X-HAIL) and Imparo directly compute the negations of certain bridge theories. Then, we regard their bridge theories $F_{h}\left(F_{x}\right)$ and $F_{i}$ as the minimal complements of a Kernel Set and a Connection theory, respectively.

Corollary 6 For each $\Gamma \in\{p, h, x, i, r\}, \Gamma$ is partially complete in generalization.

Proof of Corollary 6 Let $H$ be a maximal hypothesis wrt $\mathcal{I}_{H}$ and $F_{X}$ where $X \in$ $\{p, h, x, i\}$. By Corollary $5, H \succeq \tau\left(M\left(F_{X}\right)\right)$ holds. In case that $X=p, \tau\left(M\left(F_{p}\right)\right)$ corresponds to the bottom clause. Then, $H$ is derivable from the bottom clause by $p$. In case that $X \in\{h, x\}, F_{X}$ corresponds to $M(K S)$ for some Kernel Set $K S$. Then, $\tau\left(M\left(F_{X}\right)\right)=$ $\tau\left(M^{2}(K S)\right)$ holds. By Theorem 3, $\tau\left(M\left(F_{X}\right)\right)=\tau(\mu(K S))$ holds. Since $K S$ does not contain tautologies, $H \succeq \mu(K S)$ holds. Then, $H$ is derivable from $K S$ by $X \in\{h, x\}$. In case that $X=i, F_{i}$ corresponds to $M(C T)$ for some Connection Theory $C T$. Since $C T$ also does 
not contain tautologies, $H$ is derivable from $C T$ by $i$, just like in the above case. In case that $X=r$, by Corollary $4, H \succeq R\left(F_{r}\right)$ holds. Hence, $H$ is derivable from $R\left(F_{r}\right)$ by $r$.

Every IS-based method at least ensures the partial completeness of generalization. Progol and HAIL are incomplete in generalization by Example 15. On the other hand, it is still an open question whether or not X-HAIL and Imparo are also incomplete. The residue procedure is incomplete in generalization by Example 6. However, by Corollary 2, it becomes complete by adding the tautologies to the original bridge theory.

Our results can be applied to CF-induction in order to logically simplify its generalization procedure. Previously, it generalizes the negation of $F$ to a hypothesis $H$ using anti-entailment. As shown in Sect. 2.1, this generalization involves many non-deterministic operators that are the cause of its huge search space. By Main Theorem, it is sufficient to generalize $\tau\left(M\left(F \cup \operatorname{Taut}\left(\mathcal{I}_{H}\right)\right)\right)$ to $H$ using anti-subsumption. This simplification enables us to systematically search relevant hypotheses in the subsumption lattice bounded by $\tau\left(M\left(F \cup \operatorname{Taut}\left(\mathcal{I}_{H}\right)\right)\right)$. Indeed, the other IS-based methods developed refinement operators to efficiently explore with heuristics the lattice structure (Tamaddoni-Nezhad and Muggleton 2009). By our results, such previously proposed sophisticated techniques can be embedded in $\mathrm{CF}$-induction, while it preserves the completeness for finding hypotheses.

\section{Conclusion and future work}

This paper has shown a new form of inverse subsumption that can be embedded in a complete induction procedure. Most IE-based methods use anti-subsumption, instead of antientailment, for their generalization. However, it has not yet been clarified whether or not this logical reduction affects the completeness of generalization. For this open problem, we have shown that inverse subsumption can ensure the completeness only by adding tautologies associated with a language bias to the original bridge theory.

We have investigated the possible hypotheses obtained by each previously proposed method like Progol, HAIL, X-HAIL, Imparo and the residue procedure. As a result, we have shown that they are at least partially complete in the sense that they can derive any maximal hypotheses. The residue procedure becomes complete by simply adding tautologies to its bridge theories. In contrast, it is an open question whether or not X-HAIL and Imparo preserve the completeness of generalization. It would be fruitful to consider this question in future: if they could construct the theory obtained by adding the tautologies as another bridge theory, then they should be complete.

We have also shown that CF-induction can be logically simplified using the new form of inverse subsumption. Inverse entailment needs many non-deterministic operators like inverse resolution which cause its huge search space. This simplification enables us to focus on the search space characterized as a bounded subsumption lattice. This search space never involve inverse resolution. We intend to investigate how the search space can be reduced by the simplification in future.

Efficient implementation of inverse subsumption is an important future work. There is an efficient algorithm for enumerating the minimal hitting sets (Satoh and Uno 2002; Uno 2002). This is applicable to computing the minimal complement, and is solvable in quasipolynomial total time (Fredman and Khanchiyan 1996). However, if the induction field $\mathcal{I}_{H}$ contains many complementary literals, we need vast computational costs, since the number of tautologies in $\operatorname{Taut}\left(\mathcal{I}_{H}\right)$ becomes large. To restrict them, one may consider a closed world 
assumption or does not allow new terms that do not appear in a prior knowledge base. This issue on how to provide relevant induction fields should be addressed in future work.

It is also necessary to develop an algorithm to systematically explore the subsumption lattice bounded by the minimal complement. This issue is related to refinement operators, which have been studied in ILP. They use heuristics for guiding like compression and the description length. We emphasis that inverse subsumption ensures the completeness of generalization. Hence, it can derive hypotheses which are beyond reach for incomplete methods. In this point of view, we believe the algorithm for our approach should keep its completeness in some way. For example, it would be beneficial to target an enumerating algorithm that produces hypotheses in an incremental way.

Acknowledgements The authors would like to thank the anonymous reviewers for giving us useful and constructive comments. This research is supported by 2008-2011 JSPS Grant-in-Aid Scientific Research (A) (No. 20240016) and by 2010-2012 JSPS Grant-in-Aid Scientific Research (B) (No. 22700141).

\section{Appendix A: Proof of Theorem 3}

We first introduce the notion of minimal hitting sets to prove Theorem $3 .^{6}$

Definition 9 ((Minimal) Hitting set) Let $\Pi$ be a finite set and $\mathcal{F}$ be a subset family of $\Pi$. A finite set $E$ is a hitting set of $\mathcal{F}$ if for every $F \in \mathcal{F}, E \cap F \neq \emptyset$. A finite set $E$ is a minimal hitting set of $\mathcal{F}$ if $E$ satisfies the following two conditions:

1. $E$ is a hitting set of $\mathcal{F}$;

2. For every subset $E^{\prime} \subseteq E$, if $E^{\prime}$ is a hitting set of $\mathcal{F}$, then $E^{\prime}=E$.

It is known that minimal hitting sets satisfy the following lemma:

Lemma 4 (Uno 2002) Let $\mathcal{F}$ be a family of sets and $E$ be a hitting set of $\mathcal{F}$. $E$ is a minimal hitting set of $\mathcal{F}$ if and only if for every element $e \in E$, there is a set $F \in \mathcal{F}$ such that

$$
E \cap F=\{e\} .
$$

\section{Proof of Lemma 4}

$(\Rightarrow)$ Suppose that there is an element $e \in E$ such that $E \cap F \neq\{e\}$ for every set $F \in \mathcal{F}$. Let $E^{\prime}$ be the subset $E-\{e\}$ of $E$. Then, it holds that $E^{\prime} \cap F \neq \emptyset$ for every set $F \in \mathcal{F}$, since $E$ is a hitting set of $\mathcal{F}$ and $E \cap F \neq\{e\}$ for every $F \in \mathcal{F}$. By the second condition of Definition $9, E$ is not a minimal hitting set of $\mathcal{F}$.

$(\Leftarrow)$ Suppose that $E$ is not a minimal hitting set of $\mathcal{F}$. There is a subset $E^{\prime}$ of $E$ such that $E^{\prime}$ is a hitting set of $\mathcal{F}$. For every $F \in \mathcal{F}, E^{\prime} \cap F \neq \emptyset$ holds. There is an element $e$ in $E$ such that $e \notin E^{\prime}$. Since $E^{\prime} \subseteq E-\{e\}$, for every $F \in \mathcal{F},(E-\{e\}) \cap F \neq \emptyset$ also holds. Accordingly, $E \cap F \neq\{e\}$ holds for every $F \in \mathcal{F}$. Hence, there is an element $e \in E$ such that $E \cap F \neq\{e\}$ for every $F \in \mathcal{F}$.

\footnotetext{
${ }^{6}$ Note that the proof of Theorem 3 has been partially shown in Yamamoto et al. (2009a), which is not a journal paper. We then show the full proof in this paper.
} 
Let $S$ be a family of sets $\left\{C_{1}, \ldots, C_{n}\right\}$ where each $C_{i}(1 \leq i \leq n)$ is a finite set of ground literals $\left\{l_{i}^{1}, \ldots, l_{i}^{m_{i}}\right\}$. In the following, $\mathcal{F}(S)$ denotes the family of sets $\left\{C_{1}^{*}, C_{2}^{*}, \ldots, C_{n}^{*}\right\}$ where each $C_{i}^{*}$ is the set of literals $\left\{\neg l_{i}^{1}, \ldots, \neg l_{i}^{m_{i}}\right\}$. Let $S$ be a ground clausal theory. ${ }^{7}$ Then, $\mathcal{F}(\mathcal{F}(S))$ corresponds to $S$.

Example 16 Let $S$ be the clausal theory $\{a \vee \neg b, \neg b \vee \neg c, \neg b \vee \neg d\}$. Then, $\mathcal{F}(S)$ and $\mathcal{F}(\mathcal{F}(S))$ are represented as follows:

$$
\begin{aligned}
& \mathcal{F}(S)=\{\{\neg a, b\},\{b, c\},\{b, d\}\}, \\
& \mathcal{F}(\mathcal{F}(S))=\{\{a, \neg b\},\{\neg b, \neg c\},\{\neg b, \neg d\}\} .
\end{aligned}
$$

Given a ground clausal theory $S$, the number of minimal hitting sets of the family $\mathcal{F}(\mathrm{S})$ is finite. We denote by $M H S(S)$ the finite set of all the minimal hitting sets of $\mathcal{F}(S)$. Then, $M H S(S)$ corresponds to the minimal complement $M(S)$ as follows.

Example 17 Recall Example 16. Then, $M H S(S), \bar{S}$ and $M(S)$ are as follows:

$$
\begin{aligned}
& \operatorname{MHS}(S)=\{\{\neg a, c, d\},\{b\}\}, \\
& \bar{S}=\{\neg a \vee b, \neg a \vee b \vee d, \neg a \vee c \vee d, \neg a \vee c \vee d, b, b \vee d, b \vee c, b \vee c \vee d\}, \\
& M(S)=\{\neg a \vee c \vee d, b\} .
\end{aligned}
$$

We notice that $M H S(S)$ indeed corresponds to $M(S)$.

Lemma 5 Let $S$ be a ground clausal theory. Then $M(S)=M H S(S)$ holds.

Proof of $M H S(S) \subseteq M(S)$ Let $E$ be a minimal hitting set of $\mathcal{F}(S)$. We show $E \in \mu(\bar{S})$ since $\mu(\bar{S})=M(S)$ by the definition of minimal complements. By Lemma 4, for each literal $e_{i} \in E(1 \leq i \leq n)$, there is a set $F_{i} \in \mathcal{F}(S)$ such that $E \cap F_{i}=\left\{e_{i}\right\}$. We denote by $\mathcal{F}_{E}$ the subfamily $\left\{F_{1}, \ldots, F_{n}\right\}$ of $\mathcal{F}(S)$ where each $F_{i}$ is the above set for each literal $e_{i} \in E$. By the definition of complements, each clause in $\bar{S}$ is constructed by selecting one literal $l$ from each set in $\mathcal{F}(S)$. Since $E$ is a minimal hitting set of $\mathcal{F}(S)$, for each set $F$ in $\mathcal{F}(S)$, $E \cap F \neq \emptyset$ holds. Then, $E$ can be constructed by selecting the literal $e_{i} \in E$ from each set $F_{i} \in \mathcal{F}_{E}$ and by selecting any literal $e$ in $E \cap F$ from another set $F \in \mathcal{F}(S)-\mathcal{F}_{E}$. Hence, $E \in \bar{S}$ holds. Suppose that $E \notin \mu(\bar{S})$. Then, there is a clause $D \in \bar{S}$ such that $D \subset E$. Since $D \in \bar{S}, D$ is a hitting set of $\mathcal{F}(S)$. However, this contradicts that $E$ is a minimal hitting set of $\mathcal{F}(S)$. Therefore, $E \in \mu(\bar{S})$ holds.

Proof of $M(S) \subseteq M H S(S) \quad$ Suppose that

(*) there is a clause $D \in M(S)$ such that $D \notin M H S(S)$.

Since $D \in \mu(\bar{S})$ and $\mu(\bar{S}) \subseteq \bar{S}, D \in \bar{S}$ holds. By the definition of $\bar{S}, D$ satisfies that $C \cap D \neq$ $\emptyset$ for every $C \in \mathcal{F}(S)$. Hence, $D$ is a hitting set of $\mathcal{F}(S)$. Accordingly, there is a clause $D^{\prime}$

\footnotetext{
${ }^{7}$ Each clause in $S$ is identified with the set of literals which it contains (See Sect. 2.1).
} 
in $M H S(S)$ such that $D^{\prime} \subseteq D$. Since we assume $D \notin M H S(S), D \neq D^{\prime}$ holds. Then, we get $D^{\prime} \subset D$. Since $D^{\prime} \in M H S(S)$ and $M H S(S) \subseteq M(S)$, we have $D^{\prime} \in M(S)$. Hence, there is a clause $D^{\prime} \in M(S)$ such that $D^{\prime}$ properly subsumes the clause $D$ in $M(S)$. This contradicts the minimality of $M(S)$. Hence, the primary assumption $\left(^{*}\right)$ is false. Therefore for every clause $D \in M(S), D \in M H S(S)$ holds.

Based on Lemma 5, we consider Theorem 3 in the context of minimal hitting sets. Hereafter, we simply denote $M H S(M H S(S))$ by $M H S^{2}(S)$.

Lemma 6 Let $S$ be a ground clausal theory. Then $M_{H}^{2}(S)=\mu(S)$ holds.

Proof of $\mu(S) \subseteq M H S^{2}(S)$ We show every clause in $\mu(S)$ is a minimal hitting set of $\mathcal{F}(M H S(S))$. By the definition of $M H S(S)$, for every clause $D \in M H S(S), D$ satisfies that $D \cap C \neq \emptyset$ for every set $C \in \mathcal{F}(S)$. In other words, for every set $C \in \mathcal{F}(S), C$ satisfies that $D \cap C \neq \varnothing$ for every clause $D \in M H S(S)$. Then, every set $C \in \mathcal{F}(S)$ is a hitting set of $M H S(S)$. Let $C^{\prime}$ be the set of negations of literals in $C$. Since $C \in \mathcal{F}(S), C^{\prime} \in \mathcal{F}(\mathcal{F}(S))$ holds. Since $C$ is a hitting set of $M H S(S), C^{\prime}$ is a hitting set of $\mathcal{F}(M H S(S))$. Accordingly, every set $C \in \mathcal{F}(\mathcal{F}(S))$ is a hitting set of $\mathcal{F}(M H S(S))$. Since the family $\mathcal{F}(\mathcal{F}(S))$ corresponds to $S$, it holds that every clause $C \in S$ is a hitting set of $\mathcal{F}(M H S(S))$. Since $\mu(S) \subseteq S$, every clause $C \in \mu(S)$ is a hitting set of $\mathcal{F}(M H S(S))$.

Suppose that there is a clause $C \in \mu(S)$ such that $C$ is not a minimal hitting set of $\mathcal{F}(M H S(S))$. Then,

(*) there is a literal $l \in C$ such that $C-\{l\}$ is a hitting set of $\mathcal{F}(\operatorname{MHS}(S))$.

For every clause $C_{i} \in \mu(S)$, if $C_{i} \neq C$ then there is a literal $l_{i} \in C_{i}$ such that $l_{i} \notin C$. We then consider those literals $E=\left\{l_{1}, l_{2}, \ldots, l_{n}\right\}$ where each $l_{i}$ is a literal of $C_{i} \in \mu(S)-\{C\}$ such that $l_{i}$ is not included in $C$. Note that $E \cap C=\emptyset$ holds. On the other hand, for any literal $l \in C$, it holds that $(E \cup\{l\}) \cap D \neq \emptyset$ for every clause $D \in \mu(S)$. Hence, $E \cup\{l\}$ is a hitting set of $\mu(S)$. Accordingly, $E \cup\{l\}$ is also a hitting set of $S$. Then, there is a minimal hitting set $E^{\prime}$ of $S$ such that $E^{\prime} \subseteq E \cup\{l\}$. Since $M H S(S)$ is the set of minimal hitting sets of $\mathcal{F}(S), \mathcal{F}(M H S(S))$ corresponds to the set of minimal hitting sets of $\mathcal{F}(\mathcal{F}(S))$. Since $\mathcal{F}(\mathcal{F}(S))=S, \mathcal{F}(M H S(S))$ is the set of minimal hitting sets of $S$. Hence, we have $E^{\prime} \in \mathcal{F}(M H S(S))$. Since $E^{\prime} \subseteq E \cup\{l\}$, it holds that $E^{\prime} \cap(C-\{l\}) \subseteq(E \cup\{l\}) \cap(C-\{l\})$. Since $E \cap C=\emptyset,(E \cup\{l\}) \cap(C-\{l\})=\emptyset$ holds. Accordingly, we have $E^{\prime} \cap(C-\{l\})=\emptyset$. However, this contradicts that $C-\{l\}$ is a hitting set of $\mathcal{F}(M H S(S))$, since $E^{\prime} \in \mathcal{F}(M H S(S))$. Then, the assumption (*) is false. Hence, every clause $C$ in $\mu(S)$ is a minimal hitting set of $\mathcal{F}(M H S(S))$.

Proof of $M H S^{2}(S) \subseteq \mu(S)$ Let $D$ be a clause in $M H S^{2}(S)$. Suppose that there is a clause $C \in \mu(S)$ such that $C \subset D$. Since $\mu(S) \subseteq M H S^{2}(S), C \in M H S^{2}(S)$ holds. This contradicts with the minimality of $M H S^{2}(S)$. Hence, for every clause $C \in \mu(S), C \not \subset D$. In other words, for ever clause $C \in \mu(S), C=D$ or $C \nsubseteq D$. Suppose that

(*) for any clause $C \in \mu(S), C \neq D$ holds.

Then, $C \nsubseteq D$ holds. Accordingly, for every clause $C_{i} \in \mu(S)$, there is a literal $l_{i} \in C_{i}$ such that $l_{i} \notin D$. We consider the finite set $E=\left\{l_{1}, l_{2}, \ldots, l_{n}\right\}$ where each $l_{i}$ is the above literal for each $C_{i} \in \mu(S)$. Note that $E \cap D=\emptyset$. On the other hand, the intersection of $E$ and any clause in $\mu(S)$ is not empty. Hence, $E$ is a hitting set of $\mu(S)$. Accordingly, $E$ is a hitting set 
of $S$. Then, there is a minimal hitting set $E^{\prime}$ of $S$ such that $E^{\prime} \subseteq E$. Since $M H S(S)$ is the set of minimal hitting sets of $\mathcal{F}(S), \mathcal{F}(M H S(S))$ corresponds to the set of minimal hitting sets of $\mathcal{F}(\mathcal{F}(S))$. Since $\mathcal{F}(\mathcal{F}(S))=S, \mathcal{F}(M H S(S))$ is the set of minimal hitting sets of $S$. Hence, we have $E^{\prime} \in \mathcal{F}(M H S(S))$. Since $E^{\prime} \subseteq E$ and $E \cap D=\emptyset, E^{\prime} \cap D=\emptyset$ holds. Note that since $D \in M H S^{2}(S), D$ is a minimal hitting set of $\mathcal{F}(M H S(S))$. However this contradicts that $E^{\prime} \cap D=\emptyset$, since $E^{\prime} \in \mathcal{F}(M H S(S))$. Then, the assumption (*) is false. Hence, there is a clause $C \in \mu(S)$ such that $C=D$. Therefore, $D \in \mu(S)$ holds.

Using Lemma 5 and Lemma 6, Theorem 3 is proved as follows:

Proof of Theorem 3 By Lemma 5, $M^{2}(S)=M H S^{2}(S)$ holds. By Lemma 6, $M H S^{2}(S)=$ $\mu(S)$ holds. Hence, $M^{2}(S)=\mu(S)$ holds.

\section{Appendix B: Proof of Theorem 4}

We introduce the following deductive operators for proving this theorem:

Definition 10 (Deductive operators (Yamamoto 2003)) Let $S$ and $T$ be clausal theories. Then $T$ is directly-derivable from $S$ if $T$ is obtained from $S$ by one of the following three operators:

1. (resolution) $T=S \cup\{C\}$, where $C$ is a resolvent of two clauses $D_{1}, D_{2} \in S$.

2. (subsumption) $T=S \cup\{C\}$, where $C$ is subsumed by some clause $D \in S$.

3. (weakening) $T=S-\{D\}$ for some clause $D \in S$.

We write $S \vdash_{r} T, S \vdash_{s} T, S \vdash_{w} T$ to denote that $T$ is directly derivable from $S$ by resolution, subsumption, weakening, respectively. $\vdash_{X}^{*}$ is the reflexive and transitive closure of $\vdash_{X}$, where $X$ is one of the symbols $r, s, w$. Alternatively, $S \vdash_{X}^{*} T$ if $T$ follows from $S$ by application of zero or more $\vdash_{X}$.

Let $S$ and $T$ be clausal theories such that $S$ and $T$ contains no tautologies and $S \models T$. Then, $T$ can be generated from $S$ with a concatenation of those operators, represented by the following lemma:

Lemma 7 (Yamamoto et al. 2008) Let $S$ and $T$ be clausal theories without any tautologies. If $S \models T$, then there are two clausal theories $U$ and $V$ such that

$$
S \vdash_{r}^{*} U \vdash_{s}^{*} V \vdash_{w}^{*} T
$$

Proof of Lemma 7 Let $T=\left\{C_{1}, \ldots, C_{n}\right\}$. Then $S \models C_{i}$ for each clause $C_{i}$ in $T$. By the Subsumption Theorem there is a derivation $R_{1}^{i}, \ldots, R_{m_{i}}^{i}$ from $S$ of a clause $R_{m_{i}}^{i}$ that subsumes $C_{i}$. Hence, it is sufficient to let $U=S \cup\left\{R_{j}^{i}: 1 \leq i \leq n, 1 \leq j \leq m_{i}\right\}$ and $V=U \cup T$.

Using Lemma 7, we obtain the following lemma that allows tautologies to be included in $S$ and $T$ : 


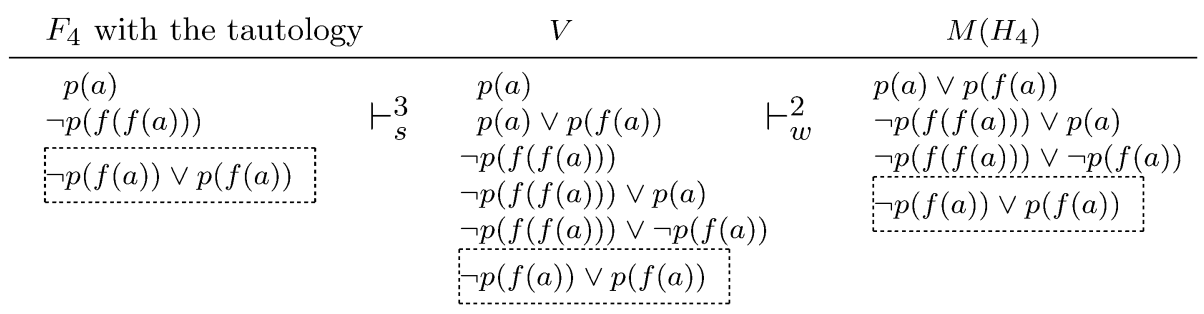

Fig. 3 A derivation from $F_{4}$ to $M\left(H_{4}\right)$ in Example 8

Lemma 8 Let $S$ and $T$ be ground clausal theories such that $S \models T$ and for every tautology $D \in T$, there is a clause $C \in S$ such that $C \succeq D$. Then there are two ground clausal theories $U$ and $V$ such that

$$
S \vdash_{r}^{*} U \vdash_{s}^{*} V \vdash_{w}^{*} T \text {. }
$$

Proof of Lemma 8 We denote the two sets of tautologies in $S$ and $T$ by Taut and Taut $_{T}$, respectively. Since $S$ theory-subsumes $\operatorname{Taut}_{T}$, there is a ground clausal theory $V_{t}$ such that $S \vdash_{s}^{*} V_{t} \vdash_{w}^{*}$ Taut $_{T}$. By Lemma 7, there are ground clausal theories $U^{\prime}$ and $V^{\prime}$ such that $S-$ Taut $_{S} \vdash_{r}^{*} U^{\prime} \vdash_{s}^{*} V^{\prime} \vdash_{w}^{*} T-$ Taut $_{T}$. Hence, we get

$$
S \vdash_{r}^{*} U^{\prime} \cup \text { Taut }_{S} \vdash_{s}^{*} V^{\prime} \cup V_{t} \vdash_{w}^{*} T \text {. }
$$

Example 18 Firstly, we recall the bridge theory $F_{4}$ and the hypothesis $H_{4}$ in Example 8. Note that $M\left(H_{4}\right)$ contains the tautology $\neg p(f(a)) \vee p(f(a))$, though $F_{4}$ does not. Then, $M\left(H_{4}\right)$ is obtained from $F_{4}$ with the tautology (See the dotted surrounding parts) using the subsumption and weakening operators in Fig. 3.

Secondly, we recall Example 12. Let $F_{5}$ be the same bridge theory in Example 12 and $H_{g_{5}}$ be the ground hypothesis as follows:

$$
H_{g_{5}}=\{\operatorname{arc}(b, c), \operatorname{arc}(b, c) \supset \operatorname{path}(b, c)\} .
$$

Note that $H_{g_{5}}$ consists of ground instances from the target hypothesis $H_{5}$ in Example 12 and $F_{5} \models M\left(H_{g_{5}}\right) . M\left(H_{g_{5}}\right)$ is as follows:

$$
M\left(H_{g_{5}}\right)=\{\neg \operatorname{arc}(b, c) \vee \neg \operatorname{path}(b, c), \neg \operatorname{arc}(b, c) \vee \operatorname{arc}(b, c)\} .
$$

$M\left(H_{g_{5}}\right)$ contains the tautology $\neg \operatorname{arc}(b, c) \vee \operatorname{arc}(b, c)$, though $F_{5}$ does not. Then, $M\left(H_{g_{5}}\right)$ is obtained from $F_{5}$ with the tautology using the three: resolution, subsumption and weakening operators in Fig. 4.

Based on Lemma 8, we prove Theorem 4 by showing that $\tau(M(T)) \succeq \tau(M(S))$ when $S \vdash_{X} T$ holds for each symbol $X \in\{r, s, w\}$.

Lemma 9 Let $S$ and $T$ be two ground clausal theories such that $S \vdash_{r} T$. Then,

$$
\tau(M(T)) \succeq \tau(M(S)) .
$$

Proof of Lemma 9 Since $S \vdash_{r} T, T$ is written as $S \cup\{C\}$ where $C$ is a resolvent of two clauses $C_{1}$ and $C_{2}$ in $S$. Since $C_{1}$ and $C_{2}$ are ground, the resolvent $C$ is written as $\left(C_{1}-\right.$ 


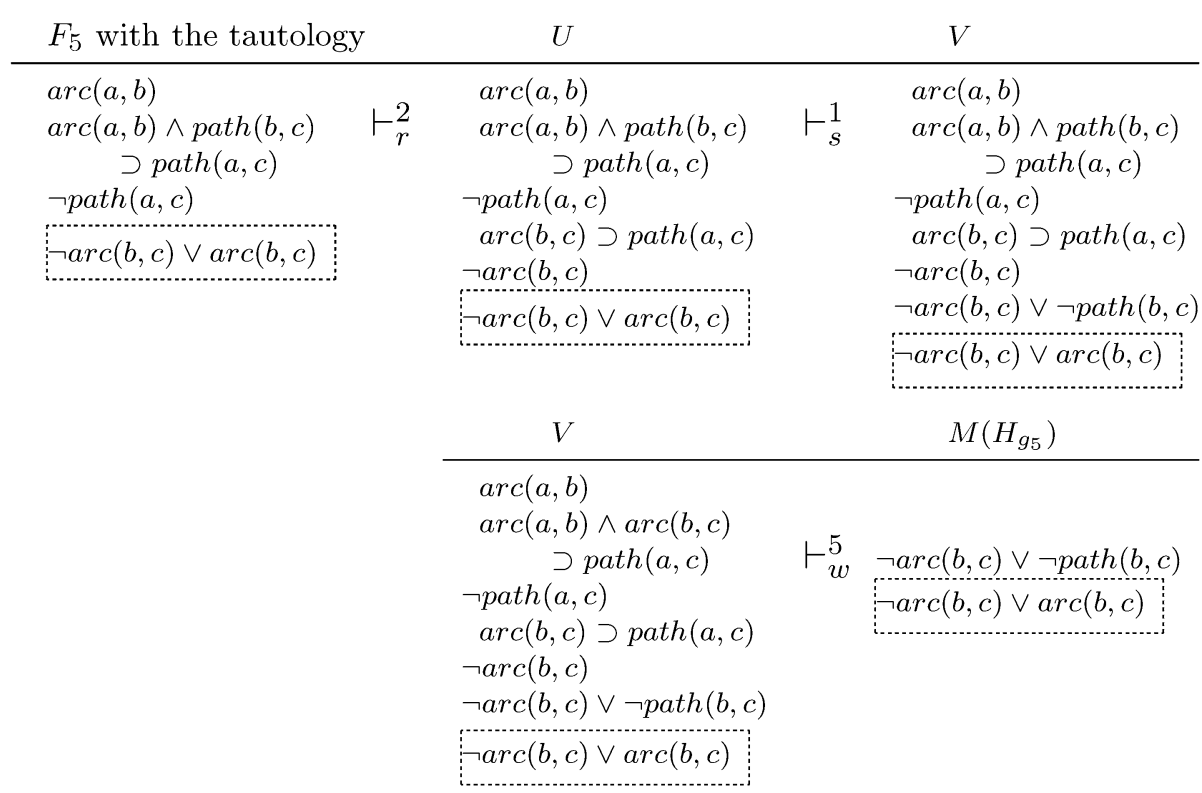

Fig. 4 A derivation from $F_{5}$ to $M\left(H_{g_{5}}\right)$ in Example 12

$\{l\}) \cup\left(C_{2}-\{\neg l\}\right)$ for some literal $l$ in $C_{1}$. Let $C^{\prime}, C_{1}^{\prime}$ and $C_{2}^{\prime}$ be three sets such that $C^{\prime}, C_{1}^{\prime}$ and $C_{2}^{\prime}$ consist of the negations of literals in $C, C_{1}$ and $C_{2}$. Since $C_{1}, C_{2} \in S, C_{1}^{\prime}$ and $C_{2}^{\prime}$ are included in $\mathcal{F}(S)$, and $C^{\prime}$ is in $\mathcal{F}(S \cup\{C\})$. Let $D$ be a clause in $\tau(M H S(S))$. Since $D$ is a minimal hitting set of $\mathcal{F}(S), D \cap C_{1}^{\prime} \neq \varnothing$ and $D \cap C_{2}^{\prime} \neq \emptyset$ hold. Suppose that

\section{(*) $\quad D$ is not a hitting set of $\mathcal{F}(S \cup\{C\})$.}

Since $C^{\prime} \in \mathcal{F}(S \cup\{C\}), D \cap C^{\prime}=\emptyset$ should hold. Since $C^{\prime}=\left(C_{1}^{\prime}-\{\neg l\}\right) \cup\left(C_{2}^{\prime}-\{l\}\right)$, $D \cap C^{\prime}=\left(\left(C_{1}^{\prime}-\{\neg l\}\right) \cap D\right) \cup\left(\left(C_{2}^{\prime}-\{l\}\right) \cap D\right)$ holds. Since $D \cap C^{\prime}=\emptyset$, it holds that $\left(C_{1}^{\prime}-\right.$ $\{\neg l\}) \cap D=\emptyset$ and $\left(C_{2}^{\prime}-\{l\}\right) \cap D=\emptyset$. Since $D \cap C_{1}^{\prime} \neq \emptyset$ and $D \cap C_{2}^{\prime} \neq \emptyset$, we obtain that $D \cap\{\neg l\} \neq \emptyset$ and $D \cap\{l\} \neq \emptyset$. Then, $D$ has complementary literals $\neg l$ and $l$. It contradicts that $D$ is not a tautology, since $D \in \tau(M H S(S))$. Thus, the assumption (*) is false. Hence, $D$ is a hitting set of $\mathcal{F}(S \cup\{C\})$. Accordingly, there is a clause $E \in M H S(S \cup\{C\})$ such that $E \succeq D$. Since $D$ is not a tautology, $E$ is also not. Then, $E \in \tau(M H S(S \cup\{C\}))$ holds. By Lemma 5, we have $\tau(M H S(S))=\tau(M(S))$ and $\tau(M H S(S \cup\{C\}))=\tau(M(S \cup\{C\}))$. Accordingly, we have $D \in \tau(M(S))$ and $E \in \tau(M(S \cup\{C\}))$. Therefore, for each clause $D \in \tau(M(S))$, there is a clause $E \in \tau(M(S \cup\{C\}))$ such that $E \succeq D$.

Lemma 10 Let $S$ and $T$ be two ground clausal theories such that $S \vdash_{s} T$. Then,

$$
\tau(M(T)) \succeq \tau(M(S)) .
$$

Proof of Lemma 10 Since $S \vdash_{s} T, T$ is written as $S \cup\{C\}$ where $C$ is a clause such that $D \succeq$ $C$ for some clause $D \in S$. Since $D$ and $C$ are ground, $D \subseteq C$ holds. Let $C^{\prime}$ and $D^{\prime}$ be two sets such that $C^{\prime}$ and $D^{\prime}$ consist of the negations of literals in $C$ and $D$, respectively. Since $D \in S, D^{\prime}$ is included in $\mathcal{F}(S)$, and $C^{\prime}$ is in $\mathcal{F}(S \cup\{C\})$. Let $E$ be a clause in $\tau(M H S(S))$. Since $E$ is a minimal hitting set of $\mathcal{F}(S), E \cap D^{\prime} \neq \emptyset$ holds. Since $D \subseteq C, D^{\prime} \subseteq C^{\prime}$ holds. 
Accordingly, $E \cap C^{\prime} \neq \emptyset$ holds. Since $C^{\prime} \in \mathcal{F}(S \cup\{C\}), E$ is a hitting set of $\mathcal{F}(S \cup\{C\})$. Then, there is a clause $E^{\prime} \in M H S(S \cup\{C\})$ such that $E^{\prime} \succeq E$. Since $E \in \tau(M H S(S)), E$ is not a tautology, and $E^{\prime}$ is also not. Hence, $E^{\prime} \in \tau(M H S(S \cup\{C\}))$ holds. By Lemma 5, we have $\tau(M H S(S))=\tau(M(S))$ and $\tau(M H S(S \cup\{C\}))=\tau(M(S \cup\{C\}))$. Therefore, for each clause $E \in \tau(M(S))$, there is a clause $E^{\prime} \in \tau(M(S \cup\{C\}))$ such that $E^{\prime} \succeq E$.

Lemma 11 Let $S$ and $T$ be two ground clausal theories such that $S \vdash{ }_{w} T$. Then,

$$
\tau(M(T)) \succeq \tau(M(S)) .
$$

Proof or Lemma 11 Since $S \vdash_{w} T, T$ is written as $S-\{C\}$ where $C$ is a clause in $S$. Let $E$ be a clause in $\tau(M H S(S))$. $E$ is a minimal hitting set of $\mathcal{F}(S)$. Since $T \subset S, \mathcal{F}(T) \subset \mathcal{F}(S)$ holds. Then, $E$ is a hitting set of $\mathcal{F}(T)$. Hence, there is a clause $E^{\prime} \in M H S(T)$ such that $E^{\prime} \succeq E$. Since $E$ is not a tautology, $E^{\prime}$ is also not a tautology, that is, $E^{\prime} \in \tau(M H S(T))$ holds. By Lemma 5, we have $\tau(M H S(S))=\tau(M(S))$ and $\tau(M H S(T))=\tau(M(S))$. Therefore, for each clause $E \in \tau(M(S))$, there is a clause $E^{\prime} \in \tau(M(T))$ such that $E^{\prime} \succeq E$.

Using Lemmas 8, 9, 10 and 11, Theorem 4 is proved as follows:

Proof of Theorem 4 By Lemma 8, there are two ground clausal theories $U$ and $V$ such that

$$
S \vdash_{r}^{*} U \vdash_{s}^{*} V \vdash_{w}^{*} T .
$$

By Lemma 9, $\tau(M(U)) \succeq \tau(M(S))$ holds. By Lemma 10, $\tau(M(V)) \succeq \tau(M(U))$ holds. By Lemma 11, $\tau(M(T)) \succeq \tau(M(V))$ holds. Hence, the following formula holds:

$$
\tau(M(T)) \succeq \tau(M(V)) \succeq \tau(M(U)) \succeq \tau(M(S)) .
$$

\section{Appendix C: Proofs of Theorem 1 and Lemma 1}

Using theoretical results in the paper, we prove Theorem 1 and Lemma 1.

Proof of Theorem 1 By Herbrand's Theorem, there is a finite subset $S^{\prime}$ of ground instances from $S$ such that $S^{\prime} \models T$. By Theorem 4, $\tau(M(T)) \succeq \tau\left(M\left(S^{\prime}\right)\right)$ holds. By Lemma 3, it holds that $\tau(M(T))=\mu(R(T))$ and $\tau\left(M\left(S^{\prime}\right)\right)=\mu\left(R\left(S^{\prime}\right)\right)$. Then, $\mu(R(T)) \succeq \mu\left(R\left(S^{\prime}\right)\right)$ holds. Since $R(T) \succeq \mu(R(T))$ and $\mu\left(R\left(S^{\prime}\right)\right) \succeq R\left(S^{\prime}\right), R(T) \succeq R\left(S^{\prime}\right)$ holds.

Proof of Lemma 1 Since $T \subseteq S, S \vdash_{w}^{*} T$ holds. By Lemma 11, $\tau(M(T)) \succeq \tau(M(S))$ holds. By Lemma 3, $\mu(R(T)) \succeq \mu(R(S))$ holds. Hence, we have $R(T) \succeq R(S)$.

\section{References}

Badea, L., \& Stanciu, M. (1999). Refinement operators can be (weakly) perfect. In LNAI: Vol. 1634. Proceedings of the 9th international workshop on inductive logic programming (pp. 21-32). Berlin: Springer.

Bratko, I. (1999). Refining complete hypotheses in ILP. In LNAI: Vol. 1634. Proceedings of the 9th international workshop on inductive logic programming (pp. 44-55). Berlin: Springer.

Chang, C. L., \& Lee, R. C. T. (1973). Symbolic logic and mechanical theorem proving. New York: Academic Press.

De Raedt, L. (1997). Logical setting for concept-learning. Artificial Intelligence, 95, 187-201. 
Fredman, M., \& Khanchiyan, L. (1996). On the complexity of dualization of monotone disjunctive normal forms. Journal of Algorithms, 28, 618-628.

Flach, P. A. (1996). Rationality postulates for induction. In Proceedings of the 6th international conference on theoretical aspects of rationality and knowledge (pp. 267-281).

Inoue, K. (2004). Induction as consequence finding. Machine Learning, 55(2), 109-135.

Kimber, T., Broda, K., \& Russo, A. (2009). Induction on failure: learning connected Horn theories. In LNCS: Vol. 5753. Proceedings of the 10th international conference on logic programming and nonmonotonic reasoning (pp. 169-181). Berlin: Springer.

Lee, C. T. (1967). A completeness theorem and computer program for finding theorems. Ph.D. thesis, Department of Electrical Engineering and Computer Science, University of California, Berkeley, CA.

Muggleton, S. H. (1995). Inverse entailment and Progol. New Generation Computing, 13, 245-286.

Muggleton, S. H., \& Buntine, W. L. (1988). Machine invention of first-order predicates by inverting resolution. In Proceedings of the 5th international conference on machine learning (pp. 339-352).

Muggleton, S. H., \& De Raedt, L. (1994). Inductive logic programming: theory and methods. The Journal of Logic Programming, 19/20, 629-679.

Nienhuys-Cheng, S., \& De Wolf, R. (1997). LNCS: Vol. 1228. Foundations of inductive logic programming. Berlin: Springer.

Ray, O. (2009). Nonmonotonic abductive inductive learning. Journal of Applied Logic, 7(3), 329-340.

Ray, O., \& Inoue, K. (2008). Mode directed inverse entailment for full clausal theories. In LNCS: Vol. 4894. Proceedings of the 17th international conference on inductive logic programming (pp. 225-238). Berlin: Springer.

Ray, O., Broda, K., \& Russo, A. M. (2003). Hybrid abductive inductive learning. In LNCS: Vol. 2835. Proceedings of the 13th international conference on inductive logic programming (pp. 311-328). Berlin: Springer.

Riguzzi, F. (2005). Two results regarding refinement operators. In Late-breaking paper proceedings of the 15th international conference on inductive logic programming (pp. 53-58).

Satoh, K., \& Uno, T. (2002). Enumerating maximal frequent sets using irredundant dualization. In LNCS: Vol. 2843. Proceedings of the 6th international conference on discovery science (pp. 256-268). Berlin: Springer.

Tamaddoni-Nezhad, A., \& Muggleton, S. H. (2009). The lattice structure and refinement operators for the hypothesis space bounded by a bottom clause. Machine Learning, 76, 37-72.

Uno, T. (2002). A practical fast algorithm for enumerating minimal set coverings. IPSJ SIG Notes, 2002(29), 9-16.

Westergaard, S. L., Oliveira, A. P., Bro, C., Olsson, L., \& Nielsen, J. (2006). A systems biology approach to study glucose repression in the yeast Saccharomyces cerevisiae. Biotechnology and Bioengineering, 96, $134-145$.

Yamamoto, A. (2003). Hypothesis finding based on upward refinement of residue hypotheses. Theoretical Computer Science, 298, 5-19.

Yamamoto, Y., Ray, O., \& Inoue, K. (2008). Towards a logical reconstruction of CF-induction. In LNCS: Vol. 4914. New frontiers in artificial intelligence (pp. 330-343). Berlin: Springer.

Yamamoto, Y., Inoue, K., \& Iwanuma, K. (2009a). Hypothesis enumeration by CF-induction. In Proceedings of the 6th workshop on learning with logics and logics for learning (pp. 80-87).

Yamamoto, Y., Inoue, K., \& Doncescu, A. (2009b). Integrating abduction and induction in biological inference using CF-induction. In H. Lodhi \& S. Muggleton (Eds.), Elements of computational systems biology (pp. 213-234). Chap. 9. 